DRAFT VERSION AUGUST 27, 2018

Preprint typeset using L LTEX style emulateapj v. 04/20/08

\title{
A LOCAL BASELINE OF THE BLACK HOLE MASS SCALING RELATIONS FOR ACTIVE GALAXIES. III. THE $M_{\mathrm{BH}}-\sigma$ RELATION
}

\author{
Vardha N. Bennert ${ }^{1}$, Tommaso Treu ${ }^{2}$, Matthew W. Auger ${ }^{3}$, Maren Cosens $^{1}$, Daeseong Park $^{4}$, RebecCa Rosen $^{1,5}$, \\ Chelsea E. Harris ${ }^{6}$, Matthew A. MalKan ${ }^{7}$, Jong-HaK Woo $^{8}$ \\ Draft version August 27, 2018
}

\begin{abstract}
We create a baseline of the black hole $(\mathrm{BH})$ mass $\left(M_{\mathrm{BH}}\right)$ - stellar-velocity dispersion $(\sigma)$ relation for active galaxies, using a sample of 66 local $(0.02<z<0.09)$ Seyfert-1 galaxies, selected from the Sloan Digital Sky Survey (SDSS). Analysis of SDSS images yields AGN luminosities free of host-galaxy contamination, and morphological classification. 51/66 galaxies have spiral morphology. 28 bulges have Sérsic index $n<2$ and are considered candidate pseudo bulges, with eight being definite pseudo bulges based on multiple classification criteria met. Only 4/66 galaxies show signs of interaction/merging. High signal-to-noise ratio Keck spectra provide the width of the broad $\mathrm{H} \beta$ emission line free of FeII emission and stellar absorption. AGN luminosity and $\mathrm{H} \beta$ line widths are used to estimate $M_{\mathrm{BH}}$. The Keck-based spatially-resolved kinematics is used to determine stellar-velocity dispersion within the spheroid effective radius $\left(\sigma_{\text {spat,reff }}\right)$. We find that $\sigma$ can vary on average by up to $40 \%$ across definitions commonly used in the literature, emphasizing the importance of using self-consistent definitions in comparisons and evolutionary studies. The $M_{\mathrm{BH}}{ }^{-} \sigma$ relation for our Seyfert1 galaxy sample has the same intercept and scatter as that of reverberation-mapped AGNs as well as that of quiescent galaxies, consistent with the hypothesis that our single epoch $M_{\mathrm{BH}}$ estimator and sample selection do not introduce significant biases. Barred galaxies, merging galaxies, and those hosting pseudo bulges do not represent outliers in the $M_{\mathrm{BH}^{-}} \sigma$ relation. This is in contrast with previous work, although no firm conclusion can be drawn on this matter due to the small sample size and limited resolution of the SDSS images.
\end{abstract}

Subject headings: accretion, accretion disks — black hole physics — galaxies: active — galaxies: evolution — quasars: general

\section{INTRODUCTION}

The discovery of relations between the mass of the central supermassive black hole (BH) and its host galaxy properties such as spheroid luminosity $L_{\text {sph }}$ (Kormendy \& Richstone 1995), spheroid mass $M_{\mathrm{sph}}$ (e.g., Magorrian et al. 1998), and spheroid stellar velocity dispersion $\sigma$ (e.g., Gebhardt et al. 2000; Ferrarese \& Merritt 2000) has sparked a flood of observational studies pertaining both to the local Universe (e.g., Merritt \& Ferrarese 2001; Tremaine et al. 2002; Marconi \& Hunt 2003; Häring \& Rix 2004; Ferrarese \& Ford 2005; Greene \& Ho 2006; Graham 2007; Gültekin et al. 2009; Bennert et al. 2011a; Kormendy \& Bender 2011; Kormendy et al. 2011; Sani et al. 2011; McConnell et al. 2011; Graham et al. 2011; Beifiori et al. 2012; Graham \& Scott 2013) and cosmic history (e.g., Treu et al. 2004, 2007; Peng et al. 2006a,b; Woo et al. 2006, 2008; Salviander et al. 2007; Riechers et al. 2009;

\footnotetext{
${ }^{1}$ Physics Department, California Polytechnic State University, San Luis Obispo, CA 93407, USA; vbennert@calpoly.edu; mcosens@ calpoly.edu

${ }^{2}$ Department of Physics, University of California, Santa Barbara, CA 93106, USA; tt@physics.ucsb.edu; Sloan Fellow, Packard Fellow; present address: Department of Physics and Astronomy, University of California, Los Angeles, CA 90095, USA

${ }^{3}$ Institute of Astronomy, Madingley Road, Cambridge CB3 OHA, UK; mauger@ast.cam.ac.uk

${ }^{4}$ National Astronomical Observatories, Chinese Academy of Sciences, Beijing 100012, China; daeseong.park@ nao.cas.cn

${ }^{5}$ Present address: AstroCamp, Idyllwild, CA 92549, USA; rosenrr@prodigy.net

${ }^{6}$ Lawrence Berkeley National Laboratory, Berkeley, California 94720, USA; ChelseaHarris@lbl.gov

${ }^{7}$ Department of Physics and Astronomy, University of California, Los Angeles, CA 90095, USA; malkan@astro.ucla.edu

${ }^{8}$ Department of Astronomy, Seoul National University, Korea; woo@astro.snu.ac.kr
}

Jahnke et al. 2009; Bennert et al. 2010; Decarli et al. 2010; Merloni et al. 2010; Bennert et al. 2011b); for a recent review see Kormendy \& Ho (2013). In particular the evolution with redshift of these correlations constrains theoretical interpretations and provides important insights into their origin (e.g., Croton 2006; Robertson et al. 2006; Hopkins et al. 2007), by probing whether BHs and their host galaxies are constantly on tight correlations through a feedback mechanism that controls their mutual growth (e.g., Kauffmann \& Haehnelt 2000; Volonteri et al. 2003; Ciotti \& Ostriker 2007; Hopkins et al. 2008), or whether the local relations are an end product of the more dramatic and stochastic process of galaxy merging in the hierarchical assembly of $M_{\mathrm{BH}}$ and stellar mass (e.g., Peng 2007; Jahnke \& Maccio 2011).

Measuring $M_{\mathrm{BH}}$ based on spatially resolving the BH's gravitational sphere of influence via stellar kinematics (e.g., van der Marel et al. 1998; Gebhardt et al. 2000), gaseous kinematics (e.g., Ferrarese et al. 1996; Marconi et al. 2001) or maser emission (e.g., Herrnstein et al. 2005; Kuo et al. 2011) is restricted to galaxies in the local Universe. The only way to probe the evolution of the scaling relations is to rely on active galaxies, thought to represent an integral phase in the evolution of galaxies during which the $\mathrm{BH}$ is growing through accretion, resulting in the luminous galaxy center known as Active Galactic Nucleus (AGN). For AGNs at cosmological distances, $M_{\mathrm{BH}}$ is estimated through application of the "virial method" (e.g., Wandel et al. 1999). In this method, it is assumed that gas clouds in the broad-line region (BLR) orbiting the $\mathrm{BH}$ in close proximity follow the gravitational field of the $\mathrm{BH}$. In a time-consuming process called reverberation mapping (e.g., Wandel et al. 1999; Kaspi et al. 2000, 2005; Bentz et al. 2006, 2013), the delayed response of broad emission 
lines to changes in the AGN continuum is translated into a size of the BLR. When combined with the width of the broad emission lines (i.e., velocity of the gas clouds), the mass of the $\mathrm{BH}$ can be estimated, making use of the "virial coefficient" that depends on the gas kinematics and geometry. Assuming that broad-line AGNs and quiescent galaxies follow the same $M_{\mathrm{BH}}-\sigma$ relation (as probed in several studies, e.g., Woo et al. 2013, and references therein), this coefficient has been traditionally obtained by matching their $M_{\mathrm{BH}}-\sigma$ relations (e.g., Onken et al. 2004; Greene \& Ho 2006; Woo et al. 2010; Park et al. 2012; Woo et al. 2013, 2015). More recently, by modeling reverberation-mapped data directly and constraining geometry and kinematics of the BLR, $M_{\mathrm{BH}}$ has been estimated for individual objects independent of a virial coefficient (Brewer et al. 2011; Pancoast et al. 2011, 2012, 2014). A secondary method called the "single-epoch method" makes use of an empirical correlation found from reverberation mapping that directly relates the BLR size to the AGN continuum luminosity, to allow the estimation of $M_{\mathrm{BH}}$ from a single spectrum (Vestergaard 2002; Woo \& Urry 2002; Vestergaard \& Peterson 2006; McGill et al. 2008).

While the majority of evolutionary studies point toward a scenario in which $\mathrm{BH}$ growth precedes spheroid assembly (e.g., Walter et al. 2004; Treu et al. 2004; Woo et al. 2006; Shields et al. 2006; McLure et al. 2006; Peng et al. 2006a,b; Treu et al. 2007; Salviander et al. 2007; Weiss et al. 2007; Riechers et al. 2008; Woo et al. 2008; Riechers et al. 2009; Gu et al. 2009; Jahnke et al. 2009; Decarli et al. 2010; Merloni et al. 2010; Bennert et al. 2010, 2011b), no consensus has been reached on the interpretation of the evolutionary studies (see e.g., Schramm \& Silverman 2013; Volonteri \& Stark 2011; Schulze \& Wisotzki 2014, especially concerning the role of scatter, observational bias, and selection effects) and, ultimately, the origin of the $M_{\mathrm{BH}}$ scaling relations. A key toward understanding the $M_{\mathrm{BH}}$ scaling relations may lie in understanding the local relations for active galaxies and systematic effects in the analysis. First, all conclusions about evolution of these relations hinge on understanding the slope and scatter of local relations, especially those involving broad-line AGNs - the class of objects targeted by high-redshift studies, by necessity. Second, while reverberation-mapped AGNs benefit from smaller $M_{\mathrm{BH}}$ errors, their selection based on sufficient AGN variability may introduce biases (e.g., Woo et al. 2013, and references therein). Third, investigating the dependence of scaling relations on additional parameters, such as the amount of nuclear activity and the detailed properties of the host galaxies, is vital to understanding the physical origins of galaxies. For example, while the spheroid has been traditionally identified as the fundamental driver for $M_{\mathrm{BH}}$, there have been studies that point towards tighter correlations of $M_{\mathrm{BH}}$ with the total host-galaxy light or stellar mass (Bennert et al. 2010, 2011b; Jahnke et al. 2009; Läsker et al. 2014). A related open question is the role of pseudo bulges. Late-type galaxies are often known to host pseudo bulges, characterized by nearly exponential light profiles, ongoing star formation or starbursts, and nuclear bars. It is generally believed that they have evolved secularly through dissipative processes rather than mergers (Courteau et al. 1996; Kormendy \& Kennicutt 2004). Conversely, classical bulges are thought of as centrally concentrated, mostly red and quiescent, merger-induced systems. It is unclear how BHs would grow within pseudo bulges and how their masses could be related: some authors find that pseudo bulges correlate with $M_{\mathrm{BH}}$ (e.g., Kormendy 2001; Gu et al. 2009), while others propose either the op- posite (Hu 2008; Greene et al. 2010; Kormendy \& Bender 2011) or at least that both the $M_{\mathrm{BH}}-\sigma$ and $M_{\mathrm{BH}}-L_{\mathrm{sph}}$ relations are not obeyed simultaneously (Greene et al. 2008; Nowak et al. 2010).

We here present the results of a program aimed at addressing these questions by building upon a robust and unique baseline of $\sim 100$ local $(0.02 \leq z \leq 0.09)$ Seyfert-1 galaxies selected from the Sloan Digital Sky Survey (SDSS) $\left(M_{\mathrm{BH}}>\right.$ $10^{7} \mathrm{M}_{\odot}$ ) for the study of the $M_{\mathrm{BH}}$ scaling relations. The homogeneous selection of our sample based on emission lines is disjoint from the reverberation-mapped AGNs and allows us to probe selection effects in the reverberation-mapped AGN sample which serves as a $M_{\mathrm{BH}}$ calibrator for the entire Universe. Moreover, our selection is similar to high-redshift samples. Combining high-quality long-slit Keck/LRIS spectra with archival multi-filter SDSS images yields four different fundamental scaling relations. Results for a pilot sample of 25 objects have been presented by the first paper in the series (Bennert et al. 2011a, hereafter Paper I). Spatially-resolved $\sigma$ measurements for the full sample have been published by Harris et al. (2012)[hereafter Paper II].

In this paper, we focus on the $M_{\mathrm{BH}^{-}} \sigma$ relation. We have obtained high $\mathrm{S} / \mathrm{N}$ spatially-resolved long-slit spectra and measured both aperture $\sigma$ as well as spatially resolved $\sigma$ (Paper II). Here, we derive spatially resolved $\sigma$ within the bulge effective radius and compare it to different definitions of stellar velocity dispersion used in the literature.

The paper is organized as follows. We summarize sample selection, observations, and data reduction in Section 2. Section 3 describes the derived quantities, such as host-galaxy properties derived from surface photometry, stellar velocity dispersion, and $M_{\mathrm{BH}}$. In Section 4, we describe our final sample as well as comparison samples drawn from literature, consisting of local quiescent galaxies and reverberation-mapped AGNs. We present and discuss our results in Section 5. We conclude with a summary in Section 6. In Appendix A, we show fits to the broad $\mathrm{H} \beta$ emission line for a total of $79 \mathrm{ob}-$ jects for which we measured $M_{\mathrm{BH}}$. Appendix B summarizes notes for a few individual objects.

Throughout the paper, we assume a Hubble constant of $H_{0}$ $=70 \mathrm{~km} \mathrm{~s}^{-1} \mathrm{Mpc}^{-1}, \Omega_{\Lambda}=0.7$ and $\Omega_{\mathrm{M}}=0.3$.

\section{SAMPLE SELECTION, OBSERVATIONS, AND DATA REDUCTION}

Sample selection, observations, and data reduction were described in detail in Paper I \& II of the series, and are only summarized here briefly, for convenience. Details for the full sample of 103 objects are listed in Paper II (Tables 1\&2).

The sample was selected from the SDSS Data Release (DR) 6 following these criteria: (i) $M_{\mathrm{BH}}>10^{7} M_{\odot}$ as estimated based on optical luminosity and Full-Width-at-HalfMaximum (FWHM) of the broad $\mathrm{H} \beta$ line; (ii) redshift range $0.02<z<0.09$ to measure stellar kinematics via the CaII triplet line in the optical and to ensure that the objects are well resolved. A total of 103 objects were observed between 2009 January and 2010 March with the Low Resolution Imaging Spectrometer (LRIS) at Keck I using a $1^{\prime \prime}$ wide long slit, aligned with the host galaxy major axis as determined from SDSS ("expPhi_r"). The D560 dichroic (for data taken in 2009) or the D680 dichroic (for data taken in 2010) was used, the $600 / 4000$ grism in the blue, and the $831 / 8200$ grating in the red with central wavelength $8950 \AA$, resulting in an instrumental resolution of $\sim 90 \mathrm{~km} \mathrm{~s}^{-1}$ in the blue and $\sim 45 \mathrm{~km} \mathrm{~s}^{-1}$ 
in the red. A table with sample and observation details can be found in Paper II (Table 1).

The data were reduced following standard reduction steps including bias subtraction, flat fielding, and cosmic-ray rejection. Wavelengths were calibrated using arc lamps in the blue spectrum and sky emission lines in the red spectrum. Telluric absorption correction and relative flux calibration was performed using AOV Hipparcos stars.

All objects were covered by the VLA FIRST (Faint Images of the Radio Sky at Twenty-cm) survey ${ }^{9}$, but only 32 have counterparts within a radius of $5^{\prime \prime}$. Out of these, 21 are listed in Rafter et al. (2009) with only two being radio-loud. Thus, the majority of our objects are radio-quiet.

\section{DERIVED QUANTITIES}

To derive surface photometry, stellar-velocity dispersion, and $M_{\mathrm{BH}}$, we followed the same procedures as outlined in Paper I \& II. We here briefly summarize the procedure and results.

\subsection{Surface Photometry}

In Paper I, we described in detail an image analysis code "Surface Photometry and Structural Modeling of Imaging Data" (SPASMOID) designed to allow for simultaneous fitting of multi-filter images with arbitrary constraints between the parameters in each band (Bennert et al. 2011a,b). This joint multi-wavelength analysis enables a much more powerful disentanglement of the nuclear and host-galaxy components than using single-band imaging alone (e.g., using GALFIT, Peng et al. 2002). The bluer bands provide a robust measurement of the normalisation of the nuclear flux while the redder data exploit the more favorable contrast between the AGN and the host galaxy to constrain the morphological structure of the latter. The approach of simultaneously using structural and photometric information is most successful for imaging of AGN hosts given the presence of a bright AGN point source. SPASMOID's reliance on a Markov Chain Monte-Carlo (MCMC) technique also provides realistic uncertainties and the ability to explore covariances between various model parameters.

We use SPASMOID to perform surface-brightness photometry on the SDSS images, simultaneously fitting the AGN by a point-spread function (PSF) and the host galaxy by a combination of spheroid (Sérsic with free index $n$, in a range between 0.7 and 4.7), and if present, disk (exponential profile), and bar (Sérsic with index $n=0.5$, i.e., a Gaussian). The results given in Tables 1-2 correspond to the maximum a posteriori (MAP) values. Note that this approach differs slightly from Paper I, in which we fitted the spheroid with a single de Vaucouleurs (1948) profile. We thus here included all 25 objects from Paper I again and ran SPASMOID on the full sample of 103 objects. From the final sample, 11 objects were omitted due to either image defects, bright nearby stars complicating the fit, or no reliable fit achieved.

We use the Sérsic index to distinguish between classical bulges and pseudo bulges (see Section 5.1). The spheroid radius is used to determine the stellar velocity dispersion within that effective radius (see 3.2). The PSF g'-band magnitude is corrected for Galactic extinction (subtracting the SDSS DR7 "extinction_g"' column), and then extrapolated to $5100 \AA$, assuming a power law of the form $f_{\nu} \propto \nu^{\alpha}$ with $\alpha=-0.5$. The re-

\footnotetext{
${ }^{9}$ See VizieR Online Data Catalog, 8071 (Becker et al. 2003)
}

sulting AGN luminosity free of host-galaxy contribution (except potential dust attenuation) is used for $M_{\mathrm{BH}}$ measurements (see 3.3). In the subsequent papers of the series (V. N. Bennert et al. 2015, in prep.), we will discuss luminosity and stellar masses of the different components when deriving the remaining $M_{\mathrm{BH}}$-scaling relations.

\subsection{Stellar-velocity Dispersion and Spatially-resolved Kinematics}

From the full sample of 103 objects, the spectra of 21 objects did not yield a robust measurement of the stellar kinematics, due to dominating AGN flux and high redshift (12 objects) or problems with the instrument ( 9 objects), so our final kinematic sample consists of 82 objects (see Paper II, Table 2 ). While the exclusion of $10 \%$ of objects with faint galaxies compared to the AGN can in principle introduce a systematic effect in our sample, we consider this effect negligible, given the overall sample size.

For the majority of objects, broad nuclear FeII emission $(\sim 5150-5350 \AA)$ is present and interferes with the measurements of both $\sigma$ in the MgIb range and broad $\mathrm{H} \beta$ width. Thus, for those objects, a set of IZw1 templates (varying width and strength) and a featureless AGN continuum were fitted simultaneously and subtracted.

Stellar-velocity dispersion $\sigma$ was measured from three different spectra regions: around $\mathrm{CaH} \& \mathrm{~K} \lambda \lambda 3969,3934$ (hereafter CaHK), around the $\mathrm{Mg} \mathrm{Ib} \lambda \lambda \lambda 5167,5173,5184$ (hereafter MgIb) lines and around CaII $\lambda \lambda \lambda 8498,8542,8662$ (hereafter CaT). $\sigma$ measurements were obtained from a Pythonbased code described in detail in Paper I \& II. In short, it is based on the algorithm by van der Marel (1994), fitting a linear combination of Gaussian-broadened $\left(30-500 \mathrm{~km} \mathrm{~s}^{-1}\right)$ template spectra ( $\mathrm{G}$ and $\mathrm{K}$ giants of various temperatures as well as spectra of $\mathrm{A} 0$ and $\mathrm{F} 2$ giants from the Indo-US survey) and a polynomial continuum using a MCMC routine (with the best derived $\sigma$ measurements corresponding to the MAP values). Telluric and AGN emission lines were masked and thus excluded from the fit.

In Paper II, $\sigma$ measurements were derived for both aperture and spatially-resolved spectra, i.e., as a function of distance from the center. The extracted spatially-resolved spectra were used to determine the velocity dispersion within the spheroid effective radius $\sigma_{\text {spat, reff }}$, free from broadening due to a rotating disk component. (Note that this assumes that the spheroid component dominates the velocity dispersion within the spheroid effective radius, and that contributions from bar and disk are negligible in comparison.) To do so, we calculate the velocity dispersion within the spheroid effective radius as determined from the surface photometry:

$$
\sigma_{\text {spat,reff }}^{2}=\frac{\int_{-R_{\text {eff }}}^{\mathrm{R}_{\text {eff }}}\left(\sigma_{\text {spat }}^{2}(r)+v_{\text {spat }}^{2}(r)\right) \cdot I(r) \cdot r \cdot d r}{\int_{-R_{\text {eff }}}^{\mathrm{R}_{\text {eff }}} I(r) \cdot r \cdot d r}
$$

with $I(r)=I(\mathrm{reff}) \cdot \exp \left(-\kappa_{\mathrm{n}} \cdot\left[\left(\mathrm{r} / \mathrm{r}_{\mathrm{reff}}\right)^{1 / \mathrm{n}}-1\right]\right)$ the surface brightness of the spheroid fitted as a Sérsic profile. Here, $v_{\text {spat }}$ is the rotational component of the spheroid. We approximated $\kappa_{n}=1.9992 n-0.3271$ (valid for $0.5<n<10$, Capaccioli 1989; Prugniel \& Simien 1997). (Note that $n, r_{\text {reff }}$, and $I$ (reff are taken from the image analysis.) Since stellar velocity dispersions were measured for spectra on both sides of the center, along the major axis, we integrate from " $-\mathrm{R}_{\text {eff }}$ " to " $+\mathrm{R}_{\text {eff }}$ ". 
A spline function is used to interpolate over the appropriate radial range, since the $\sigma_{\text {spat }}$ measurements are discrete. In Section 5.2, we discuss other $\sigma$ definitions used in the literature and compare them with the fiducial value used throughout this Paper from Eq. 1.

\subsection{Black Hole Mass}

We measure the second moment of the broad $\mathrm{H} \beta$ emission line from the central blue Keck/LRIS spectrum (1 squarearcsecond in size), using the IDL-based code implemented by Park et al. (2015), allowing for a multi-component spectral decomposition of the $\mathrm{H} \beta$ region. Here, we briefly summarize the procedure. First, we model and subtract the observed continuum by simultaneously fitting the pseudo-continuum, which consists of the AGN featureless power-law continuum, the AGN Fe II emission template from Boroson \& Green (1992), and the host-galaxy starlight templates from the IndoUS spectral library (Valdes et al. 2004), in the emission-line free windows of $4430-4770 \AA$ and $5080-5450 \AA$. Second, we model the continuum-subtracted $\mathrm{H} \beta$ emission-line region by simultaneously fitting Gauss-Hermite series (van der Marel \& Franx 1993; McGill et al. 2008; Woo et al. 2006) to the broad and narrow $\mathrm{H} \beta$ emission lines and the [O III] $\lambda \lambda 4959,5007$ narrow emission lines and fitting Gaussian functions to the broad and narrow He II $\lambda 4686$ emission lines (when blended with the broad $\mathrm{H} \beta$ component; see Section 3.1 of Park et al. 2015 for details). The final fits are shown in Figs. A1-A2.

From the resulting fit, the second moment of the broad $\mathrm{H} \beta$ component $\left(\sigma_{H \beta}\right)$ is combined with the $5100 \AA$ AGN luminosity derived from surface photometry to estimate $M_{\mathrm{BH}}$ :

$$
\begin{aligned}
\log M_{\mathrm{BH}}=0.71+6.849+2 \log \left(\frac{\sigma_{\mathrm{H}_{\beta}}}{1000 \mathrm{~km} \mathrm{~s}^{-1}}\right) \\
+0.549 \log \left(\frac{\lambda L_{5100}}{10^{44} \mathrm{erg} \mathrm{s}^{-1}}\right)
\end{aligned}
$$

This equation is derived from adopting the most recent broadline region (BLR) radius-luminosity relation (Bentz et al. 2013)[Table 14, Clean2+ExtCorr] and a virial factor of $\log f=$ 0.71 (Park et al. 2012; Woo et al. 2013). The results are given in Tables 1-2 for a sample of 79 objects (see next paragraph). We assume a nominal uncertainty of the BH masses measured via the virial method of 0.4 dex (Vestergaard \& Peterson 2006).

Note that from the full sample of 103 objects presented in Paper II, six objects showed only a broad $\mathrm{H} \alpha$ line in the SDSS spectrum and no broad $\mathrm{H} \beta$ line in either the SDSS or Keck spectra. While we can still estimate $M_{\mathrm{BH}}$ from the broad $\mathrm{H} \alpha$ line in the SDSS spectrum, we decided to exclude them from the sample, for consistent $M_{\mathrm{BH}}$ measurements. An additional eight objects showed broad $\mathrm{H} \alpha$ and $\mathrm{H} \beta$ lines in the SDSS spectrum, but did not reveal any broad $\mathrm{H} \beta$ line in the Keck spectrum. We excluded these objects here as well, and our $M_{\mathrm{BH}}$ sample is thus comprised of 79 objects. However, we will discuss them individually in an upcoming paper (V. N. Bennert et al. 2015, in prep.). This upcoming paper will also include a direct comparison between the SDSS spectrum and the Keck spectrum, to study any broad-line variability. $57 \mathrm{ob}-$ jects are included in the BH mass function study by Greene \& Ho (2007) with $\mathrm{BH}$ masses derived from the broad $\mathrm{H} \alpha$ line and luminosity in the SDSS spectra, with an overall good agreement in the mass measurements.

\section{FINAL SAMPLE AND COMPARISON SAMPLES}

While a sample of 103 objects was observed at Keck, not all properties could be determined for all objects (see Section 3 ). Taking into account the overlap between the measurements of surface photometry, stellar velocity dispersion and $M_{\mathrm{BH}}$, our final sample for the $M_{\mathrm{BH}}-\sigma$ relation consists of 66 objects (see Tables 1-2).

We compare our sample with the compilation of $M_{\mathrm{BH}}$ and $\sigma$ for quiescent galaxies (McConnell \& Ma 2013, 72 objects) as well as reverberation-mapped AGNs (Woo et al. 2015, 29 objects; adopting the same virial factor as for our sample; $\log f=0.71)$. In Table 4, we additionally compare with the recent compilation of $M_{\mathrm{BH}}$ and $\sigma$ for quiescent galaxies by Kormendy \& Ho (2013) (51 objects; pseudo bulges and mergers excluded). Our results do not change depending on what comparison sample we use. We discuss below the effects of different $\sigma$ definitions used in the literature, including these comparison samples. Unfortunately, none of the literature uses the definition that we consider the most robust in this paper (see discussion below).

Note that while for the quiescent galaxies, $\mathrm{BH}$ masses have been derived from direct dynamical measurements, the $\mathrm{BH}$ masses for active galaxies are calibrated masses either from reverberation mapping or from the virial method. 
TABLE 1

SAMPLE AND DeRIVED QuANTITIES

\begin{tabular}{|c|c|c|c|c|c|c|c|c|c|c|c|}
\hline $\begin{array}{l}\text { Object } \\
\text { (1) }\end{array}$ & $\begin{array}{l}\text { R.A. } \\
\text { (J2000) } \\
\text { (2) }\end{array}$ & $\begin{array}{l}\text { Decl. } \\
\text { (J2000) } \\
\text { (3) }\end{array}$ & (4) & $\begin{array}{c}\sigma_{\text {spat,reff }} \\
\left(\mathrm{km} \mathrm{s}^{-1}\right) \\
(5)\end{array}$ & $\begin{array}{c}r_{\text {eff,sph }} \\
\text { (") } \\
\text { (6) }\end{array}$ & $\begin{array}{c}\sigma_{H \beta} \\
\left(\mathrm{km} \mathrm{s}^{-1}\right) \\
(7)\end{array}$ & $\begin{array}{c}\lambda L_{5100} \\
\left(10^{44} \mathrm{erg} \mathrm{s}^{-1}\right) \\
(8)\end{array}$ & $\begin{array}{c}\log M_{\mathrm{BH}} / M_{\odot} \\
\text { (9) }\end{array}$ & $\begin{array}{l}\text { Host } \\
\text { (10) }\end{array}$ & $\begin{array}{c}\text { Spheroid } \\
\text { (11) }\end{array}$ & $\begin{array}{c}\text { Alt. Name } \\
\text { (12) }\end{array}$ \\
\hline 0013-0951 & 001335.38 & -095120.9 & 0.0615 & 96 & 4.00 & $2111 \pm 211$ & 0.225 & 7.85 & $\mathrm{BD}$ & $\mathrm{C}$ & \\
\hline $0026+0009$ & 002621.29 & +000914.9 & 0.0600 & 172 & 1.54 & $1527 \pm 227$ & 0.025 & 7.05 & $\mathrm{BDB}$ & $\mathrm{C}$ & \\
\hline $0038+0034$ & 003847.96 & +003457.5 & 0.0805 & 127 & 1.23 & $3328 \pm 239$ & 0.208 & 8.23 & $\mathrm{BD}$ & $\mathrm{C}$ & \\
\hline $0109+0059$ & 010939.01 & +005950.4 & 0.0928 & 183 & 0.20 & $1797 \pm 268$ & 0.101 & 7.52 & $\mathrm{BD}$ & $\mathrm{C}$ & \\
\hline 0121-0102 & 012159.81 & -010224.4 & 0.0540 & 90 & 1.74 & $1742 \pm 106$ & 0.290 & 7.75 & BDB & $\mathrm{P}$ & MRK1503 \\
\hline $0150+0057$ & 015016.43 & +005701.9 & 0.0847 & 176 & 2.85 & $2057 \pm 129$ & 0.020 & 7.25 & $\mathrm{BD}$ & $\mathrm{C}$ & \\
\hline 0206-0017 & 020615.98 & -001729.1 & 0.0430 & 225 & 7.29 & $1979 \pm 185$ & 0.540 & 8.00 & $\mathrm{BD}(\mathrm{M})$ & $\mathrm{C}$ & UGC1597 \\
\hline $0212+1406$ & 021257.59 & +140610.0 & 0.0618 & 171 & 0.83 & $1586 \pm 86$ & 0.069 & 7.32 & $\mathrm{BD}$ & $\mathrm{C}$ & \\
\hline $0301+0115$ & 030144.19 & +011530.8 & 0.0747 & 99 & 1.90 & $1653 \pm 105$ & 0.155 & 7.55 & B & $\mathrm{C}$ & \\
\hline 0310-0049 & $03 \quad 1027.82$ & -004950.7 & 0.0801 & $\ldots$ & 0.20 & $1558 \pm 334$ & 1.172 & 7.98 & $\mathrm{BD}$ & $\mathrm{C}$ & \\
\hline $0336-0706$ & 033602.09 & -070617.1 & 0.0970 & 236 & 7.17 & $2403 \pm 192$ & 0.036 & 7.53 & $\mathrm{BD}$ & $\mathrm{C}$ & \\
\hline $0353-0623$ & 035301.02 & -062326.3 & 0.0760 & 175 & 1.11 & $1548 \pm 537$ & 0.160 & 7.50 & $\mathrm{BD}$ & $\mathrm{C}$ & \\
\hline $0731+4522$ & 073126.68 & +452217.4 & 0.0921 & $\ldots$ & 1.39 & $1885 \pm 134$ & 0.089 & 7.53 & $\mathrm{BD}$ & $\mathrm{C}$ & \\
\hline $0737+4244$ & 073703.28 & +424414.6 & 0.0882 & . & 2.53 & $1692 \pm 98$ & 0.141 & 7.55 & $\mathrm{BD}$ & $\mathrm{C}$ & \\
\hline $0802+3104$ & 080243.40 & +310403.3 & 0.0409 & 116 & 3.41 & $1772 \pm 185$ & 0.072 & 7.43 & $\mathrm{BD}$ & $\mathrm{C}$ & \\
\hline $0811+1739$ & $\begin{array}{llll}08 & 11 & 10.28\end{array}$ & +173943.9 & 0.0649 & 142 & 1.98 & $1520 \pm 361$ & 0.042 & 7.17 & $\mathrm{BD}$ & $\mathrm{C}$ & \\
\hline $0813+4608$ & 081319.34 & +460849.5 & 0.0540 & 122 & 0.99 & $1430 \pm 91$ & 0.048 & 7.14 & $\mathrm{BDB}$ & $\mathrm{P}$ & \\
\hline $0845+3409$ & 084556.67 & +340936.3 & 0.0655 & 123 & 1.15 & $1718 \pm 172$ & 0.064 & 7.37 & $\mathrm{BDB}$ & $\mathrm{P}$ & KUG0842+343A \\
\hline $0854+1741$ & 085439.25 & +174122.5 & 0.0654 & 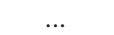 & 1.98 & $1472 \pm 269$ & 0.270 & 7.58 & BD & $\mathrm{C}$ & MRK1220 \\
\hline $0857+0528$ & 085737.77 & +052821.3 & 0.0586 & 126 & 2.22 & $1485 \pm 51$ & 0.135 & 7.42 & BD & $\mathrm{C}$ & \\
\hline $0904+5536$ & 090436.95 & +553602.5 & 0.0371 & 194 & 5.43 & $2483 \pm 36$ & 0.088 & 7.77 & $\mathrm{~B}(\mathrm{M})$ & $\mathrm{C}$ & \\
\hline $0921+1017$ & 092115.55 & +101740.9 & 0.0392 & 83 & 3.37 & $2317 \pm 286$ & 0.030 & 7.45 & $\mathrm{BD}$ & $\mathrm{C}$ & VIIIZw045 \\
\hline $0923+2254$ & 092343.00 & +225432.7 & 0.0332 & 149 & 1.43 & $1824 \pm 265$ & 0.194 & 7.69 & BDB & $\mathrm{P}$ & \\
\hline $0923+2946$ & 092319.73 & +294609.1 & 0.0625 & 142 & 3.52 & $2936 \pm 247$ & 0.019 & 7.56 & B & $\mathrm{C}$ & \\
\hline $0927+2301$ & 092718.51 & +230112.3 & 0.0262 & 196 & 13.42 & $2112 \pm 205$ & 0.005 & 6.94 & $\mathrm{BD}$ & $\mathrm{C}$ & NGC2885 \\
\hline $0932+0233$ & 093240.55 & +023332.6 & 0.0567 & 126 & 0.63 & $1814 \pm 72$ & 0.069 & 7.44 & $\mathrm{BD}$ & $\mathrm{C}$ & \\
\hline $0936+1014$ & 093641.08 & +101415.7 & 0.0600 & & 3.25 & $1995 \pm 80$ & 0.091 & 7.59 & $\mathrm{BD}$ & $\mathrm{C}$ & \\
\hline $1029+1408$ & 102925.73 & +140823.2 & 0.0608 & 185 & 2.57 & $2456 \pm 344$ & 0.133 & 7.86 & $\mathrm{BDB}$ & $\mathrm{C}$ & \\
\hline $1029+2728$ & 102901.63 & +272851.2 & 0.0377 & 112 & 3.41 & $1544 \pm 252$ & 0.014 & 6.92 & B & $\mathrm{C}$ & \\
\hline $1029+4019$ & 102946.80 & +401913.8 & 0.0672 & 166 & 1.54 & $2193 \pm 387$ & 0.093 & 7.68 & $\mathrm{BD}$ & $\mathrm{C}$ & \\
\hline $1042+0414$ & 104252.94 & +041441.1 & 0.0524 & 74 & 3.13 & $1518 \pm 102$ & 0.039 & 7.14 & $\mathrm{~B}$ & $\mathrm{C}$ & \\
\hline $1043+1105$ & 104326.47 & +110524.3 & 0.0475 & & 3.09 & $2314 \pm 28$ & 0.171 & 7.87 & B & $\mathrm{C}$ & \\
\hline $1049+2451$ & 104925.39 & +245123.7 & 0.0550 & 162 & 1.23 & $2534 \pm 135$ & 0.246 & 8.03 & BD & $\mathrm{C}$ & \\
\hline $1058+5259$ & 105828.76 & +525929.0 & 0.0676 & 122 & 0.99 & $1896 \pm 645$ & 0.075 & 7.50 & $\mathrm{BDB}$ & $\mathrm{P}$ & \\
\hline $1101+1102$ & 110101.78 & +110248.8 & 0.0355 & 197 & 8.16 & $3949 \pm 170$ & 0.068 & 8.11 & $\mathrm{BD}$ & $\mathrm{C}$ & MRK728 \\
\hline $1104+4334$ & 110456.03 & +433409.1 & 0.0493 & 87 & 1.15 & $1719 \pm 160$ & 0.016 & 7.04 & $\mathrm{BD}$ & $\mathrm{C}$ & \\
\hline $1116+4123$ & 111607.65 & +412353.2 & 0.0210 & 108 & 3.76 & $3136 \pm 384$ & 0.004 & 7.23 & $\mathrm{BD}$ & $\mathrm{C}$ & UGC6285 \\
\hline $1132+1017$ & 113249.28 & +101747.4 & 0.0440 & & 1.94 & $1900 \pm 86$ & 0.049 & 7.40 & BDB & $\mathrm{C}$ & IC2921 \\
\hline $1137+4826$ & 113704.17 & +482659.2 & 0.0541 & 155 & 1.07 & $1606 \pm 92$ & 0.006 & 6.74 & B & $\mathrm{C}$ & \\
\hline $1143+5941$ & 114344.30 & +594112.4 & 0.0629 & 122 & 3.13 & $1790 \pm 128$ & 0.099 & 7.51 & $\mathrm{BD}$ & $\mathrm{C}$ & \\
\hline $1144+3653$ & 114429.88 & +365308.5 & 0.0380 & 168 & 1.39 & $2933 \pm 205$ & 0.041 & 7.73 & BDB & $\mathrm{P}$ & KUG1141+371 \\
\hline $1145+5547$ & 114545.18 & $\begin{array}{r}+554759.6 \\
\end{array}$ & 0.0534 & 118 & 1.31 & $1837 \pm 208$ & 0.027 & 7.22 & BDB & $\mathrm{P}$ & \\
\hline $1147+0902$ & 114755.08 & +0902 28.8 & 0.0688 & 147 & 2.61 & $2896 \pm 188$ & 0.690 & 8.39 & B & $\mathrm{C}$ & \\
\hline $1205+4959$ & 120556.01 & +495956.4 & 0.0630 & 152 & 2.02 & $2678 \pm 294$ & 0.177 & 8.00 & $\mathrm{BD}$ & $\mathrm{C}$ & \\
\hline $1210+3820$ & 121044.27 & +382010.3 & 0.0229 & 141 & 1.23 & $2831 \pm 148$ & 0.062 & 7.80 & $\mathrm{BD}$ & $\mathrm{C}$ & KUG1208+3806 \\
\hline $1216+5049$ & 121607.09 & +504930.0 & 0.0308 & 189 & 6.57 & $4487 \pm 477$ & 0.035 & 8.06 & BDB & $\mathrm{C}$ & MRK1469 \\
\hline $1223+0240$ & 122324.14 & +024044.4 & 0.0235 & 124 & 7.25 & $2306 \pm 107$ & 0.007 & 7.10 & B & $\mathrm{C}$ & MRK50 \\
\hline $1231+4504$ & 123152.04 & +450442.9 & 0.0621 & 169 & 1.23 & $1555 \pm 168$ & 0.073 & 7.32 & $\mathrm{BD}(\mathrm{M})$ & $\mathrm{C}$ & \\
\hline $1241+3722$ & 124129.42 & +372201.9 & 0.0633 & 144 & 1.43 & $1574 \pm 100$ & 0.091 & 7.38 & $\mathrm{BD}$ & $\mathrm{C}$ & \\
\hline $1246+5134$ & 124638.74 & +513455.9 & 0.0668 & 119 & 3.05 & $1141 \pm 130$ & 0.044 & 6.93 & $\mathrm{BD}$ & $\mathrm{C}$ & \\
\hline $1306+4552$ & 130619.83 & +455224.2 & 0.0507 & 114 & 2.34 & $1892 \pm 297$ & 0.018 & 7.16 & BD & $\mathrm{C}$ & \\
\hline $1307+0952$ & 130721.93 & +095209.3 & 0.0490 & & 3.21 & $1630 \pm 165$ & 0.041 & 7.22 & $\mathrm{BD}$ & $\mathrm{C}$ & \\
\hline $1312+2628$ & 131259.59 & +262824.0 & 0.0604 & 109 & 1.47 & $1572 \pm 496$ & 0.154 & 7.51 & $\mathrm{BD}$ & $\mathrm{C}$ & \\
\hline $1323+2701$ & 132310.39 & +270140.4 & 0.0559 & 124 & 0.87 & $2414 \pm 376$ & 0.026 & 7.45 & $\mathrm{BD}$ & $\mathrm{C}$ & \\
\hline $1355+3834$ & 135553.52 & +383428.5 & 0.0501 & & 2.77 & $4034 \pm 301$ & 0.097 & 8.21 & $\mathrm{~B}$ & $\mathrm{C}$ & MRK464 \\
\hline $1405-0259$ & 140514.86 & -025901.2 & 0.0541 & 125 & 0.59 & $1599 \pm 140$ & 0.020 & 7.04 & $\mathrm{BD}$ & $\mathrm{C}$ & \\
\hline $1416+0137$ & 141630.82 & +013707.9 & 0.0538 & 173 & 3.41 & $1514 \pm 233$ & 0.064 & 7.26 & BD & $\mathrm{C}$ & \\
\hline $1419+0754$ & 141908.30 & +075449.6 & 0.0558 & 215 & 4.99 & $3006 \pm 371$ & 0.116 & 8.00 & $\mathrm{BD}$ & $\mathrm{C}$ & \\
\hline $1434+4839$ & 143452.45 & +483942.8 & 0.0365 & 109 & 1.23 & $1731 \pm 85$ & 0.210 & 7.66 & BDB & $\mathrm{C}$ & NGC5683 \\
\hline $1505+0342$ & 150556.55 & +034226.3 & 0.0358 & $\ldots$ & 2.10 & $2127 \pm 139$ & 0.382 & 7.98 & $\mathrm{BD}$ & $\mathrm{C}$ & MRK1392 \\
\hline $1535+5754$ & 153552.40 & +575409.3 & 0.0304 & 110 & 4.51 & $2442 \pm 93$ & 0.287 & 8.04 & B & $\mathrm{C}$ & MRK290 \\
\hline $1543+3631$ & 154351.49 & +363136.7 & 0.0672 & 146 & 2.93 & $1820 \pm 168$ & 0.229 & 7.73 & $\mathrm{BD}$ & $\mathrm{C}$ & \\
\hline $1545+1709$ & 154507.53 & +170951.1 & 0.0481 & 163 & 1.15 & $3588 \pm 226$ & 0.070 & 8.03 & $\mathrm{BD}$ & $\mathrm{C}$ & \\
\hline $1554+3238$ & 155417.42 & +323837.6 & 0.0483 & 158 & 1.82 & $2523 \pm 159$ & 0.125 & 7.87 & $\mathrm{BD}$ & $\mathrm{C}$ & \\
\hline $1557+0830$ & 155733.13 & +083042.9 & 0.0465 & $\ldots$ & 1.62 & $2388 \pm 91$ & 0.063 & 7.66 & B & $\mathrm{C}$ & \\
\hline
\end{tabular}

NotE. - Col. (1): target ID used throughout the text (based on R.A. and declination). Col. (2): right ascension. Col. (3): declination. Col. (4): redshift from SDSS-DR7. Col. (5):

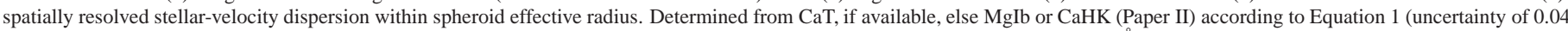

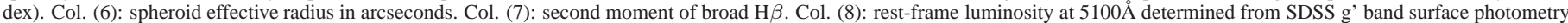

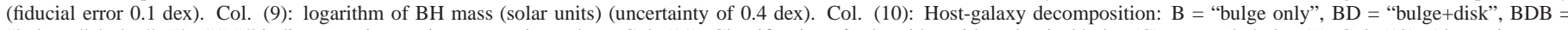

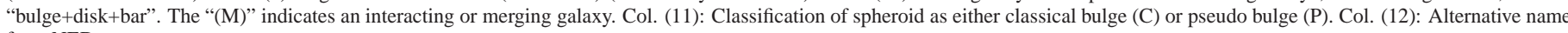
from NED. 
TABLE 2

SAMPLE AND DERIVED QuANTITIES

\begin{tabular}{|c|c|c|c|c|c|c|c|c|c|c|c|}
\hline $\begin{array}{c}\text { Object } \\
\text { (1) } \\
\end{array}$ & $\begin{array}{l}\text { R.A. } \\
(\mathrm{J} 2000) \\
(2)\end{array}$ & $\begin{array}{c}\text { Decl. } \\
(\mathrm{J} 2000) \\
(3)\end{array}$ & $\begin{array}{l}z \\
(4)\end{array}$ & $\begin{array}{c}\sigma_{\text {spat, reff }} \\
\left(\mathrm{km} \mathrm{s}^{-1}\right) \\
(5)\end{array}$ & $\begin{array}{l}r_{\text {eff,sph }} \\
\text { (") } \\
(6)\end{array}$ & $\begin{array}{c}\sigma_{H \beta} \\
\left(\mathrm{km} \mathrm{s}^{-1}\right) \\
(7)\end{array}$ & $\begin{array}{c}\lambda L_{5100} \\
\left(10^{44} \mathrm{erg} \mathrm{s}^{-1}\right) \\
(8)\end{array}$ & $\begin{array}{c}\log M_{\mathrm{BH}} / M_{\odot} \\
(9)\end{array}$ & $\begin{array}{l}\text { Host } \\
\text { (10) }\end{array}$ & $\begin{array}{c}\text { Spheroid } \\
\text { (11) }\end{array}$ & $\begin{array}{c}\text { Alt. Name } \\
\text { (12) }\end{array}$ \\
\hline $1605+3305$ & 160502.46 & +330544.8 & 0.0532 & 187 & 1.58 & $1960 \pm 272$ & 0.254 & 7.82 & B & $\mathrm{C}$ & \\
\hline $1606+3324$ & 160655.94 & +332400.3 & 0.0585 & 157 & 1.54 & $2053 \pm 80$ & 0.067 & 7.54 & BD & $\mathrm{C}$ & \\
\hline $1611+5211$ & 161156.30 & +521116.8 & 0.0409 & 116 & 1.66 & $2515 \pm 410$ & 0.056 & 7.67 & $\mathrm{BD}$ & $\mathrm{C}$ & \\
\hline $1636+4202$ & 163631.28 & +420242.5 & 0.0610 & 205 & 8.24 & $2492 \pm 230$ & 0.125 & 7.86 & $\mathrm{BD}$ & $\mathrm{P}$ & \\
\hline $1708+2153$ & 170859.15 & +215308.1 & 0.0722 & 231 & 5.86 & $2975 \pm 122$ & 0.276 & 8.20 & $\mathrm{~B}(\mathrm{M})$ & $\mathrm{C}$ & \\
\hline $2116+1102$ & 211646.33 & +110237.3 & 0.0805 & & 10.38 & $2484 \pm 42$ & 0.220 & 7.99 & $\mathrm{BD}$ & $\mathrm{C}$ & \\
\hline $2140+0025$ & 214054.55 & +002538.2 & 0.0838 & 126 & 1.90 & $1114 \pm 64$ & 0.585 & 7.52 & B & $\mathrm{C}$ & \\
\hline $2215-0036$ & 221542.29 & -003609.6 & 0.0992 & . & 5.66 & $1636 \pm 92$ & 0.202 & 7.61 & BD & $\mathrm{C}$ & \\
\hline $2221-0906$ & 222110.83 & -090622.0 & 0.0912 & 142 & 3.60 & $2375 \pm 131$ & 0.104 & 7.77 & B & $\mathrm{C}$ & \\
\hline $2222-0819$ & 222246.61 & -081943.9 & 0.0821 & 122 & 1.07 & $1799 \pm 168$ & 0.177 & 7.66 & $\mathrm{BD}$ & $\mathrm{C}$ & \\
\hline $2233+1312$ & 223338.42 & +131243.5 & 0.0934 & 193 & 1.19 & $2477 \pm 135$ & 0.368 & 8.11 & BD & $\mathrm{C}$ & \\
\hline $2254+0046$ & 225452.24 & +004631.4 & 0.0907 & & 2.73 & $989 \pm 261$ & 0.481 & 7.37 & $\mathrm{~B}(\mathrm{M})$ & $\mathrm{C}$ & \\
\hline $2327+1524$ & 232721.97 & +152437.4 & 0.0458 & 225 & 7.29 & $1924 \pm 166$ & 0.079 & 7.52 & B & $\mathrm{C}$ & \\
\hline $2351+1552$ & 235128.75 & +155259.1 & 0.0963 & 186 & 1.43 & $2974 \pm 144$ & 0.165 & 8.08 & B & $\mathrm{C}$ & \\
\hline
\end{tabular}

NOTE. - Table 1 continued. 


\section{RESULTS AND DISCUSSION}

For the discussion of our results, we only consider our final sample of 66 objects for which we have both $M_{\mathrm{BH}}$ and $\sigma$ measurements.

\subsection{Host-galaxy Morphologies}

We visually inspected the multi-filter SDSS images as well as the fits and residuals to determine the best host-galaxy decomposition. The majority of the host galaxies are classified as $\mathrm{Sa}$ or later $(51 / 66=77 \%)$ and was fitted either by a spheroid+disk decomposition (40) or spheroid+disk+bar (11). For the remaining 15 objects, a spheroid only fit was deemed sufficient. The high fraction of spiral galaxies is typical for a sample of (mostly radio-quiet) Seyfert galaxies (e.g., Hunt \& Malkan 1999, and references therein). This is consistent with the majority of objects $(\sim 60 \%)$ showing rotation curves with a maximum velocity between 100 and $200 \mathrm{~km} \mathrm{~s}^{-1}$. $6 \%$ of the sample (4/66) are merging or interacting galaxies. This is lower than for our high-redshift Seyfert galaxies $(\sim 30 \%$ at $z \simeq 0.4-0.6$ Park et al. 2015) and more comparable to quiescent galaxies in the local Universe.

Of the 79 objects in our sample, 75 are included in the morphological classification by Galaxy Zoo (Lintott et al. 2011), but for only 28 did the vote reach the necessary $80 \%$ mark to flag the morphology as either spiral or elliptical. For those, our classification agrees in the majority of cases $(82 \%)$ with the rest being classified as ellipticals by Lintott et al. (2011) while we classified them as spirals. However, we consider our classification as more robust since it also takes into accounts the fits and residuals, especially given the AGN central point source, which could lead to an overestimation of the presence of a bulge by Galaxy Zoo.

For the spiral galaxies in our sample, a little more than half of spheroid components are fitted by a Sérsic index $<2(28$ objects $=55 \%$, corresponding to $43 \%$ of the total sample) . For the rest ( 23 objects), the spheroid component is fitted by a Sérsic index $\geq 2$. On average, the Sérsic index for the spiral galaxies is $2.2 \pm 1.6$, for $n \geq 2$ bulges $3.8 \pm 0.8$, and for $n<2$ bulges $0.9 \pm 0.3$.

If we take solely the Sérsic index as an indicator for the existence of a classical vs. pseudo bulge, half of our spiral galaxies have pseudo bulges; we consider them candidate pseudo bulges. The host-galaxies of the reverberation-mapped AGNs have a similar distribution: Ho \& Kim (2014) classify 75\% as spiral galaxies with roughly half having classical bulges and half having pseudo bulges. In a bulge+disk decomposition of galaxies in SDSS, roughly $25 \%$ of galaxies with sufficient image quality to study their bulge profile shape (a total of $\sim 53,000$ ) have pseudo bulges, if we use the same criterion with Sérsic index $<2$ (Simard et al. 2011, their Fig. 15, when excluding bars with $n=0.5$ ).

However, we further follow the guidelines by Kormendy \& Ho (2013) to distinguish between classical bulges and pseudo bulges, applying the following four criteria that we can probe with our data: (i) Sérsic index $n<2$ for pseudo bulges, $n \geq 2$ for classical bulges; (ii) bulge-to-total luminosity ratios (in all four SDSS bands) $\mathrm{B} / \mathrm{T}>0.5$ for classical bulges; (iii) $v_{\text {max,reff }} / \sigma_{\text {center }}>1$ for pseudo bulges, $<1$ for classical bulges (here, $v_{\text {max,reff }}$ is the maximum velocity at the effective radius of the bulge and $\sigma_{\text {center }}$ is the stellar velocity dispersion in the center); (iv) the presence of a bar in face-on galaxies as an indicator of a pseudo bulge. To be conservative, we only classify objects as having a pseudo bulge for which at least three of the above four criteria are met. That leaves us with a total of eight spiral galaxies with a definite pseudo bulge.

\subsection{Stellar-velocity Dispersions}

The measurement of the stellar velocity dispersion profiles and rotation curves is described in detail in Paper II. In this paper, with the addition of the surface photometry parameters, we have all the necessary information to investigate the systematic uncertainties and biases related to the definition of the stellar velocity dispersion.

With this goal in mind, we carry out a systematic comparison between different definitions of the velocity dispersion parameter taken from the literature and our fiducial measurement $\left(\sigma_{\text {spat,reff }}\right.$, Eq. 1$)$. Specifically we compute velocity dispersions as average of the second moment of the velocity field, by varying the size of the aperture, by considering the difference between correcting and not correcting the velocity rotation curve for inclination, and by considering the effects of contamination by nuclear light. While not $100 \%$ exhaustive, our list of definitions includes most of the choices adopted in the literature. For example, Ferrarese \& Merritt (2000) use the second moment, integrating over one quarter of the effective radius of the galaxy and correcting the velocity for inclination. Neither Gültekin et al. (2009), McConnell \& Ma (2013), nor Kormendy \& Ho (2013) correct the velocity for inclination. However, Gültekin et al. (2009) and McConnell \& Ma (2013) use the effective radius of the galaxy, while Kormendy \& Ho (2013) use half of the effective radius of the galaxy. McConnell \& Ma (2013) additionally discuss the choice of the minimum radius and find that setting it to zero (as done by e.g., Gültekin et al. 2009) can result in $\sigma$ values smaller by $10-15 \%$, since it includes signal from within the BH gravitational sphere of influence. Thus, they instead set the minimum radius to the latter. However, for our data, we are not resolving the $\mathrm{BH}$ gravitational sphere of influence. Finally, many $\sigma$ measurements in the literature are derived from aperture spectra, such as SDSS fiber spectra or spectra of distant galaxies integrated over different aperture sizes, making a direct comparison difficult. The reverberation-mapped AGN comparison sample falls into this category (Woo et al. 2015).

Table 3 lists the results for different possible comparisons, and Fig. 1 shows three examples. Here, $\sigma_{\text {spat }, 0.5 \text { reff }}\left(\sigma_{\text {spat }, 0.25 \text { reff }}\right)$ integrates out to half (one quarter) of the effective bulge radius. $\sigma_{\text {spat,reff,sini }}$ additionally corrects the velocity for inclination, as estimated from the disk:

$$
\sigma_{\text {spat, reff,sini }}^{2}=\frac{\int_{-R_{\text {eff }}}^{R_{\text {eff }}}\left(\sigma_{\text {spat }}^{2}(r)+v_{\text {spat }}^{2} / \sin (i)^{2}(r)\right) \cdot I(r) \cdot r \cdot d r}{\int_{-R_{\text {eff }}}^{\mathrm{R}_{\text {eff }}} I(r) \cdot r \cdot d r}
$$

Again, we adopt smaller integration radii in $\sigma_{\text {spat, } 0.5 \text { reff,sini }}$ and $\sigma_{\text {spat }, 0.25 \text { reff,sini (half and one quarter of the effective bulge ra- }}$ dius, respectively). $\sigma_{\text {spat,reff,galaxy }}$ integrates out to the effective radius of the galaxy instead, or alternatively half/one quarter of that $\left(\sigma_{\text {spat }, 0.5 \text { reff,galaxy }} ; \sigma_{\text {spat }, 0.25 \text { reff,galaxy }}\right)$. It follows that $\sigma_{\text {spat,reff,galaxy,sini }}$ corrects the velocity for inclination, considering different integration limits in $\sigma_{\text {spat }, 0.5 \text { reff,galaxy,sini }}$ and $\sigma_{\text {spat }, 0.25 \text { reff,galaxy,sini. }}$ Finally, $\sigma_{\text {spat,SDSS }}$ integrates within the 1.5 " radius of the SDSS fiber. (Note that in fact our $\sigma_{\mathrm{ap}, \mathrm{SDSS}}$ corresponds to a rectangular region with $1 . " 5$ radius and $1^{\prime \prime}$ width, given the width of the long slit used.) 
The largest (average) effect on the derived $\sigma$ is the correction of the velocity for inclination, which can result in $\sigma$ measurements by on average $31 \pm 9 \%$ larger (see Fig. 1) $(43 \pm 9 \%$ in case of the galaxy effective radius), with individual objects as much as doubled. This discrepancy is reduced by considering smaller integration limits, since rotation is negligible at the center of most objects. However, such large differences are mainly due to galaxies seen close to face on, for which the velocities are highly uncertain and sometimes inflated beyond reasonably physical limits, resulting in large outliers. For example, if we impose arbitrarily that $v / \sin (i)<\sigma$ (i.e., rotation not dominant), the average ratio falls to $1.06 \pm 0.01$. Or if instead less stringent we impose that $v / \sin (i)<2 \sigma$ (e.g., pseudo bulges may be rotation dominated), the average ratio is $1.14 \pm 0.02$. Based on this source of uncertainty, we emphasize that the large difference should not be taken at face value, but more as an indication that for face-on galaxies we simply do not know the contribution of the rotational support. The intrinsic uncertainty in inclination correction for face-on objects is compounded by the fact that we do not have a good estimate of the inclination of the bulge. In the definitions of $\sigma$ above labeled as 'sini', we used the inclination of the disk component as a proxy. Given these caveats, we decided to not correct the velocities for inclination in our fiducial measurement, $\sigma_{\text {spat,reff }}($ Eq. 1), which is a common practice in the literature, including the comparison samples considered here. However, we caution that $\sigma$ might be underestimated in some cases.

When increasing the radius to the galaxy effective radius, the $\sigma$ measurement decreases on average by $1 \pm 1 \%$ (see Fig. 1). This is expected, since most objects show a decreasing stellar velocity dispersion with distance from the center (Paper II).

However, there is another effect that might counterbalance this trend: choosing a larger radius also includes more and more rotational velocities, in particular those of the disk. Depending on the viewing angle, disk rotation can lead to overestimating $\sigma$, if seen edge-on, or under-estimating $\sigma$, if seen face-on, given that the disk is kinematically cold (e.g., Woo et al. 2006, 2013, Paper I \& II). This effect can be potentially important, given the variety of host-galaxy morphologies in both local (e.g., Malkan et al. 1998; Hunt \& Malkan 2004; Kim et al. 2008; Bentz et al. 2009; Bennert et al. 2011a) and distant AGNs (Bennert et al. 2010, 2011b; Park et al. 2015). When considering the different morphologies and inclinations in our comparison between $\sigma_{\text {spat,reff }}$ and $\sigma_{\text {spat,reff,galaxy }}$, we confirm the expected trend.

Bellovary et al. (2014) use cosmological smoothed particle hydrodynamics (SPH) simulations of five disk galaxies and find that the line-of-sight effect due to galaxy orientation can affect $\sigma$ by $30 \%$ with face-on views resulting in systematically lower velocity dispersion measurements and edge-on orientations leading to higher values due to a contamination of rotating disk stars. Both fiber-based SDSS data (e.g., Greene \& Ho 2006; Shen et al. 2008) as well as aperture spectra for distant galaxies (e.g., our studies on the evolution of the $M_{\mathrm{BH}}-\sigma$ relation; Woo et al. 2006; Treu et al. 2007; Woo et al. 2008) can suffer from the added uncertainty of the effect of the disk on $\sigma$. The evolutionary studies rely on active galaxies, by necessity, and given the distance of the galaxies, stellar velocity dispersion measurements are challenging. Aperture spectra typically include the central few kpc of the galaxy (for a typical aperture of $1^{\prime \prime}=5-7 \mathrm{kpc}$ for the redshift range of $z=0.36-0.57$ covered by e.g., Woo et al. 2008), and can be contaminated by the disk kinematics, especially for Seyfert-1 galaxies chosen for these studies due to their relatively weak AGN power-law continuum. However, the evolutionary trend found by these studies is that of distant spheroids having on average smaller velocity dispersions than local ones. This effect cannot be explained by disk kinematics, since we would expect the opposite from rotational support. Thus, the offset of the distant AGNs from the local $M_{\mathrm{BH}^{-}} \sigma$ scaling relations is, if anything, underestimated.

Choosing a generic radius of $1 . \prime 5$ as the SDSS fiber results in an underestimation of $\sigma$ by $3 \pm 1 \%$ (Fig. 1). Note, however, that this difference is very sensitive to the distance of the particular objects, and the ratio between aperture size to bulge (or galaxy) effective radius. For our sample, the effective bulge radius (on average $2.9 \pm 0.3$ ) is close to the SDSS fiber size, so the difference is small.

In all the $\sigma$ definitions mentioned above, we measured $\sigma$ (and the velocity) as a function of distance from the center and integrated over it later out to the effective bulge radius (according to Eq. 1). However, this is not the same as directly integrating over the entire spectrum (out to the effective radius) and then measuring $\sigma$, since the latter will always include AGN power law and emission lines, while in the former approach the central spectrum is sometimes excluded due to AGN contamination (Paper II). Thus, we also compare our fiducial stellar velocity dispersion $\sigma_{\text {spat,reff }}$ with the corresponding value derived from aperture spectra which on average overestimates $\sigma$ by $1 \pm 2 \%$.

To conclude, these comparisons show that the choice of the exact definition of $\sigma$ can have a non-negligible effect (up to $40 \%$ ) and care needs to be taken, especially when comparing to other values in the literature. It is important to understand the effects of inclination, the rotational contribution of the disk, and the AGN contribution in the center, on the measurement of stellar velocity dispersion. We consider the value used in this paper, $\sigma_{\text {spat,reff }}(\mathrm{Eq} .1)$, the stellar velocity dispersion within the bulge effective radius derived from spatially resolved $\sigma$ and velocity measurements, the most robust measurement since it excludes contributions of disk rotation and AGN emission. Moreover, distinguishing between bulge and disk in the context of the $\mathrm{BH}$ mass scaling relations is especially important if BHs correlate only with the bulge component and not the disk, as suggested by recent studies (Kormendy et al. 2011; Kormendy \& Ho 2013).

\section{3. $M_{\mathrm{BH}^{-}} \sigma$ Relation}

In Fig. 2, we show the resulting $M_{\mathrm{BH}^{-}} \sigma$ relation, as well as the offset from the fiducial relation. Overall, our sample follows the same $M_{\mathrm{BH}}-\sigma$ relation as that of reverberation-mapped AGNs as well as that of quiescent galaxies. Our sample covers a small dynamical range in $\mathrm{BH}$ mass $\left(6.7<\log M_{\mathrm{BH}}<8.2\right)$, mainly due to the fact that we selected low-luminous Seyfert galaxies with lower mass $\mathrm{BHs}$ to enable $\sigma$ measurements. Considering the uncertainties of $M_{\mathrm{BH}}$ of 0.4 dex, we cannot independently determine the slope of the local relationship. The sample size is, however, sufficient to determine the zero point and scatter of the distribution around the local relationship, assuming a choice of the slope. Thus, when fitting a linear relation to the data of the form

$$
\log \left(M_{\mathrm{BH}} / M_{\odot}\right)=\alpha+\beta \log \left(\sigma / 200 \mathrm{~km} \mathrm{~s}^{-1}\right)
$$

we keep the value of $\beta$ fixed to the corresponding relationships of quiescent galaxies (5.64 for McConnell \& Ma 
(2013) and 4.38 for Kormendy \& Ho (2013)) or reverberation mapped AGNs (Woo et al. 2015, 3.97). The results are summarized in Table 4. Both zero point and intrinsic scatter are comparable to that of the quiescent galaxies, within the uncertainties, consistent with the hypothesis that the $M_{\mathrm{BH}}$ estimates we adopt do not introduce significant uncertainty in addition to the estimated one. Furthermore, biases based on different selection functions (reverberation mapped AGNs selected based on variability; quiescent galaxies selected based on the ability to resolve the $\mathrm{BH}$ gravitational sphere of influence; our sample selected based on $\mathrm{H} \beta$ line width) can be considered negligible.

When probing dependencies on host-galaxy morphology, we find that barred galaxies (comprising 17\% of the sample) do not lie preferentially off the $M_{\mathrm{BH}}-\sigma$ relation, in agreement with studies by Graham (e.g., 2008); Bentz et al. (e.g., 2009); Beifiori et al. (e.g., 2012) (see, however, Graham \& Li 2009). Also, neither merging galaxies (6\%) nor pseudo bulges (12\%) form particular outliers from the relation. This statement also holds true when considering all candidate pseudo bulges (i.e., objects with spheroid with Sérsic index $n<2$; a total of $43 \%$ of the sample). In fact, candidate pseudo bulges show an even smaller scatter in the relation.

While the latter is in agreement with some studies (e.g., Kormendy 2001; Gu et al. 2009), more recent studies suggest the opposite (Hu 2008; Greene et al. 2010; Kormendy \& Bender 2011; Kormendy \& Ho 2013; Ho \& Kim 2014). Pseudo bulges, characterized by nearly exponential light profiles, ongoing star formation or starbursts, and nuclear bars, are believed to have evolved secularly through dissipative processes rather than mergers (Courteau et al. 1996; Kormendy \& Kennicutt 2004), unlike their classical counterparts.

Given the sample of Seyfert-1 galaxies comprised of a majority of late-type galaxies and the small fraction of mergers, our results are consistent with secular evolution, driven by disk or bar instabilities and/or minor mergers, growing both BHs through accretion and spheroids through a re-distribution of mass from disk to bulge (e.g., Croton 2006; Parry et al. 2009; Jahnke et al. 2009; Cisternas et al. 2011; Bennert et al. 2010, 2011b; Schramm \& Silverman 2013). We can only speculate here that the smaller scatter exhibited by pseudo bulges might in fact be explained by a synchronizing effect that secular evolution has on the growth of BHs and bulges, growing both simultaneously at a small but steady rate. Major mergers, on the contrary, believed to create classical bulges (and elliptical galaxies) are a more stochastic and dramatic phenomenon with episodes of strong $\mathrm{BH}$ and bulge growth that can be out of sync due to the different time scales involved for growing $\mathrm{BH}$ and bulge in a major merger (e.g., Hopkins 2012). We cannot directly probe the latter with our data - as already mentioned, galaxies with obvious signs of interactions and mergers do not form particular outliers from the relation, but this is based on a very small sample statistics of $6 \%$ mergers.

However, we conclude with a note of caution: For one, when splitting our sample into sub-samples (such as classical versus pseudo bulges), the results suffer from small sample statistics. Second, our morphological classification relies on ground-based SDSS images with inherent limitations such as limited depth and resolution. For example, the kpcscale of bars and bulges is comparable to the spatial resolution of the SDSS images $\left(\mathrm{a} \sim 1.5^{\prime \prime}\right.$ PSF corresponds to 1.5 $\mathrm{kpc}$ at $\mathrm{z}=0.05$ ), significant given the presence of the bright point source in AGNs. The seeing in the ground-based SDSS

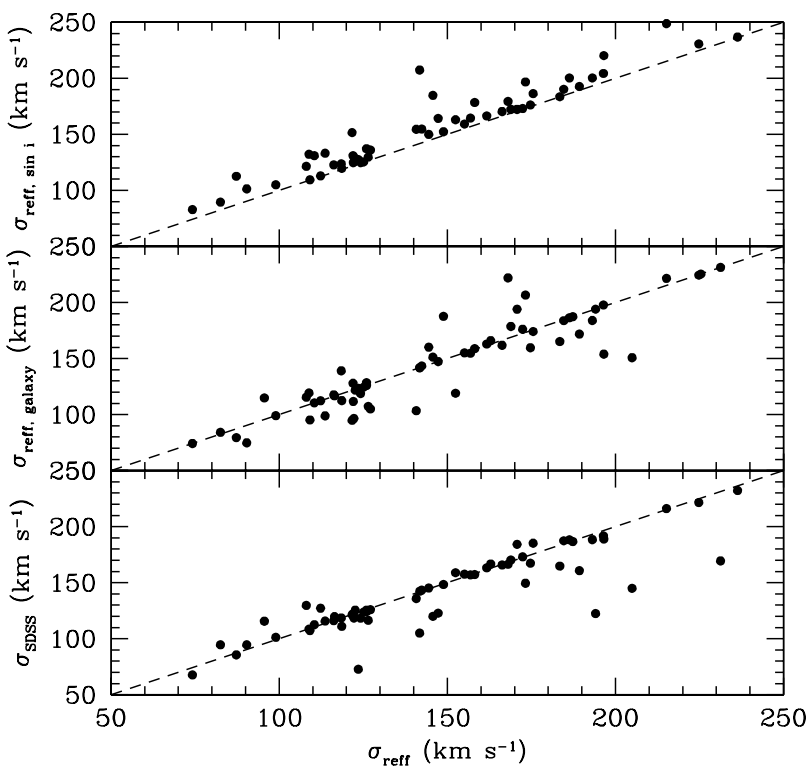

FIG. 1.- Comparison between stellar velocity dispersion as measured according to different definitions. The fiducial definition used throughout this paper, $\sigma_{\text {spat,reff, }}($ Eq. 1$)$ is shown on the x-axis, while alternative definitions based on different apertures (lower panel: 'SDSS' corresponds to 1." 5 radius; middle panel: 'galaxy' corresponds to the effective radius of the entire galaxy) or including corrections to the velocity for inclination (upper panel: 'sini'). The dashed line represents the identity.

TABLE 3

COMPARISON BETWEEN DIFFERENT DEFINITIONS FOR THE STELLAR VELOCITY DISPERSION

\begin{tabular}{|c|c|}
\hline $\begin{array}{c}\text { Ratio } \\
\text { (1) }\end{array}$ & $\begin{array}{c}\text { Mean } \\
\text { (2) }\end{array}$ \\
\hline 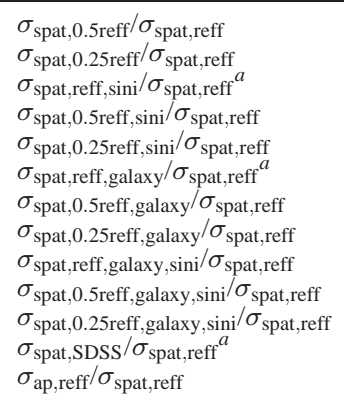 & $\begin{array}{l}0.98 \pm 0.01 \\
0.99 \pm 0.02 \\
1.31 \pm 0.09 \\
1.14 \pm 0.06 \\
1.05 \pm 0.04 \\
0.99 \pm 0.01 \\
0.96 \pm 0.01 \\
0.98 \pm 0.02 \\
1.43 \pm 0.09 \\
1.18 \pm 0.06 \\
1.07 \pm 0.04 \\
0.97 \pm 0.01 \\
1.01 \pm 0.02\end{array}$ \\
\hline \multicolumn{2}{|c|}{ 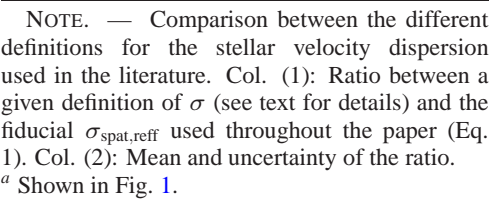 } \\
\hline
\end{tabular}

images might also cause the host galaxies to appear rounder than they actually are. Likewise, faint tidal features indicative of merger events might have been missed in these shallow images. Deeper and higher spatial-resolution images are required to further test the connection between bars and bulges and BHs. 

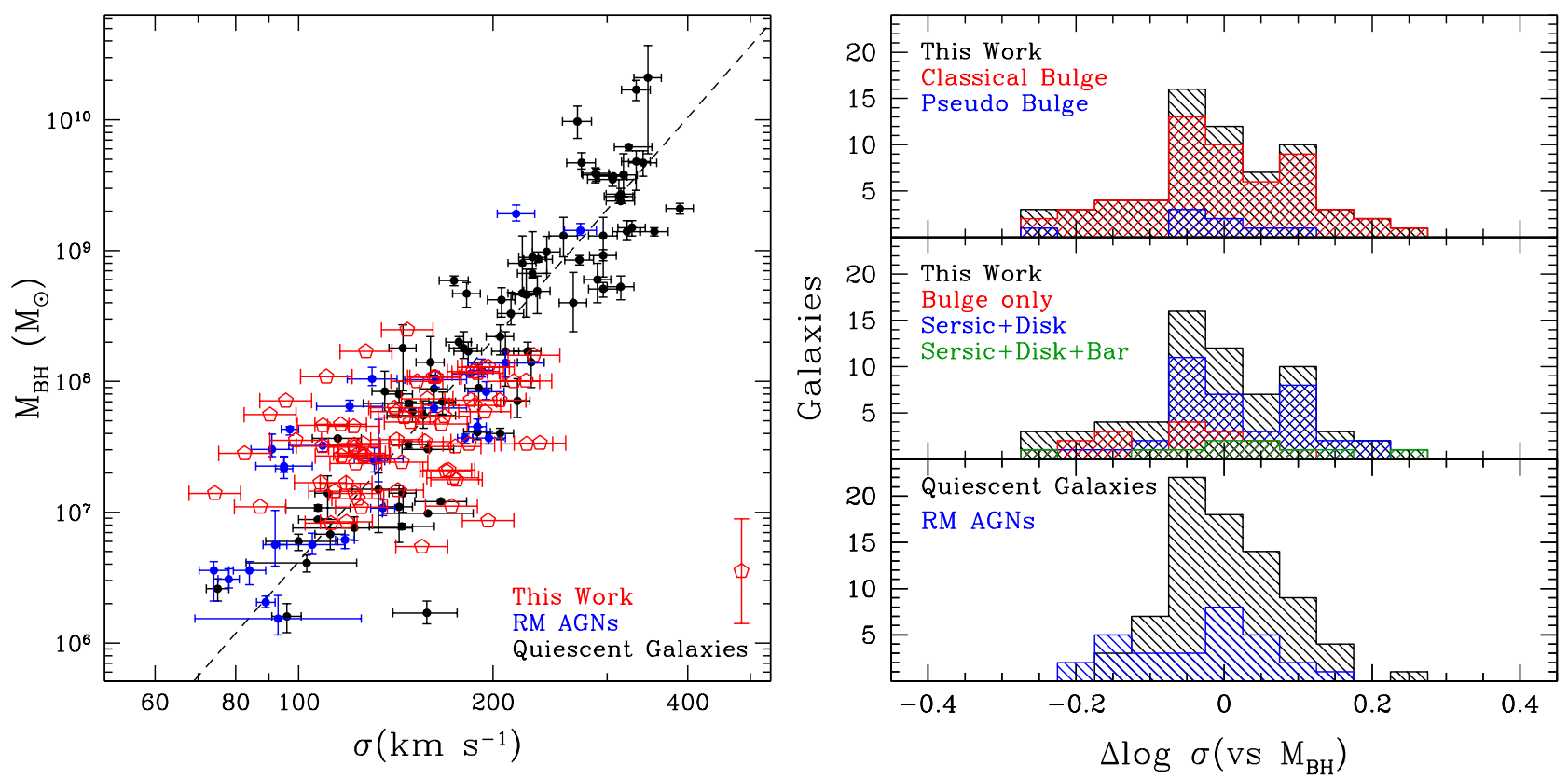

FIG. 2. $-M_{\mathrm{BH}}-\sigma$ relation. Left: $M_{\mathrm{BH}}-\sigma$ relation for our sample (red open pentagons), reverberation-mapped AGNs (blue; Woo et al. 2015), and a sample of quiescent local galaxies (black; McConnell \& Ma 2013, with the black dashed line being their best fit). The error on the BH mass for our sample is 0.4 dex and shown as a separate point with error bar in the legend, to reduce confusion of data points. For our sample, the stellar velocity dispersion was determined within the spheroid effective radius according to Equation 1. We assume a nominal uncertainty of the stellar velocity dispersion of 0.04 dex. The reverberation-mapped AGNs have $\sigma$ values derived from single apertures; $\sigma$ for the quiescent galaxies is determined similar to Equation 1, but within the galaxy effective radius (see Section 5.2 for further comparison and discussion). Right: Distribution of residuals with respect to the fiducial local $M_{\mathrm{BH}^{-}} \sigma$ relation (McConnell \& Ma 2013 , Table 4). The lower panel shows literature data (reverberation-mapped AGNs from Woo et al. (2015) in blue; quiescent galaxies from McConnell \& Ma (2013) in black), The middle panel shows our sample, the full sample in black, with different colors corresponding to different host-galaxy morphologies as indicated. The upper panel is the same as the middle panel, but distinguishing between classical and pseudo bulges.

TABLE 4

Fits TO THE LOCAL $M_{\mathrm{BH}^{-}} \sigma$ RELATIONS

\begin{tabular}{lccccc}
\hline \hline \multicolumn{1}{c}{$\begin{array}{c}\text { Sample } \\
(1)\end{array}$} & $\begin{array}{c}\alpha \\
(2)\end{array}$ & $\begin{array}{c}\beta \\
(3)\end{array}$ & $\begin{array}{c}\text { Scatter } \\
(4)\end{array}$ & $\begin{array}{c}\text { Offset } \\
(5)\end{array}$ & $\begin{array}{c}\text { Reference } \\
(6)\end{array}$ \\
\hline Quiescent Galaxies (72) & $8.32 \pm 0.05$ & $5.64 \pm 0.32$ & 0.38 & & McConnell \& Ma 2013 \\
Quiescent Galaxies (51) & $8.49 \pm 0.05$ & $4.38 \pm 0.29$ & 0.29 & & Kormendy \& Ho 2013 \\
Reverberation-mapped AGNs (29) & $8.16 \pm 0.18$ & $3.97 \pm 0.56$ & $0.41 \pm 0.05$ & & Woo et al. 2015 \\
AGNs (66) & $8.38 \pm 0.08$ & 5.64 (fixed) & $0.43 \pm 0.09$ & $-0.01 \pm 0.01$ & this paper \\
AGNs (66) & $8.20 \pm 0.06$ & 4.38 (fixed) & $0.25 \pm 0.10$ & $0.06 \pm 0.01$ & this paper \\
AGNs (66) & $8.14 \pm 0.06$ & 3.97 (fixed) & $0.19 \pm 0.10$ & $0.02 \pm 0.01$ & this paper \\
\hline
\end{tabular}

Note. - Fits to the $M_{\mathrm{BH}}-\sigma$ relation, $\log \left(M_{\mathrm{BH}} / M_{\odot}\right)=\alpha+\beta \log \left(\sigma / 200 \mathrm{~km} \mathrm{~s}^{-1}\right)$ Col. (1): Sample and sample size in parenthesis. Col. (2): Mean and uncertainty on the best fit intercept. Col. (3): Mean and uncertainty on the best fit slope. Col. (4): Mean and uncertainty on the best fit intrinsic scatter. Col. (4): Mean and uncertainty of offset from fiducial relation of either McConnell \& Ma (2013) (slope fixed to 5.64), Kormendy \& Ho (2013) (slope fixed to 4.38), or Woo et al. (2015) (slope fixed to 3.97). Col. (5): References for fit. Note that the quoted literature uses FITEXY with a uniform prior on the intrinsic scatter, so our fits assume the same.

${ }^{a}$ Relation plotted as dashed lines in Fig. 2 and used as fiducial relation when calculating residuals.

\section{SUMMARY}

To understand the origin of the scaling relations between the mass of the central supermassive $\mathrm{BH}$ and the properties of the host galaxy, studies relying on type-1 active galaxies probe the evolution of these relations. The robust determination of slope, scatter, and dependencies of a baseline consisting of a comparable sample of type-1 AGNs in the local Universe is essential to minimize biases before any conclusions about their evolution can be drawn. We here create a local baseline of the $M_{\mathrm{BH}}-$ stellar-velocity dispersion $(\sigma)$ relation for a homogeneously selected sample of 66 Seyfert- 1 galaxies in the local Universe $(0.02<z<0.09)$ selected from SDSSDR6 based on BH mass $\left(M_{\mathrm{BH}}>10^{7} M_{\odot}\right)$, Combining high S/N long-slit Keck spectra with SDSS images yields $M_{\mathrm{BH}}$ using the virial method and stellar-velocity dispersion $\left(\sigma_{\text {spat,reff }}\right)$ from spatially-resolved kinematics. Our results can be summarized as follows.

1. The majority of host galaxies (77\%) are classified as $\mathrm{Sa}$ or later with roughly one-quarter of those showing evidence for a bar. This high fraction is also reflected in prominent rotation curves with a maximum velocity of $100-200 \mathrm{~km} \mathrm{~s}^{-1}$ in the majority of kinematic measurements $(60 \%)$. The majority of spiral galaxies (28 objects; $55 \%$ ) have spheroid components with Sérsic index $n<2$. Of these candidate pseudo bulges, eight can be considered as definite, when applying the classification criteria given by Kormendy \& Ho (2013) that are measurable with our data. The minority $(6 \%)$ shows signs of merging or interacting galaxies, 
comparable to quiescent galaxies in the local Universe. These host-galaxy morphologies are typical for a sample of Seyfert-1 galaxies and suggest BH accretion being dominated by secular processes.

2. We use our spatially resolved kinematic data to reproduce various measurements of the stellar-velocity second moment, as used in the literature. The derived quantities differ significantly across definitions, in particular when considering effects of inclination, inclusion of a kinematically cold, rotating disk, and an AGN power law continuum swamping the stellar absorption lines in the center. The average differences can be as much as $40 \%$, highlighting the importance of using self-consistent definitions when comparing samples. We consider the definition of $\sigma$ used in this paper as derived from spatially resolved measurements and integrated out to the bulge effective radius the most robust value for studies of the $M_{\mathrm{BH}^{-}} \sigma$ relation, excluding disk rotation and AGN contamination.

3. Our Seyfert-1 galaxy sample follows the same $M_{\mathrm{BH}^{-}}$ $\sigma$ relation as the one of reverberation-mapped AGNs as well as that of quiescent galaxies, with the same intercept and scatter. This is consistent with the hypotheses that the uncertainty of the single epoch $M_{\mathrm{BH}}$ estimates we adopt is not significantly underestimated, and that the broad-emission line selection does not introduce a significant bias with respect to the criteria used in comparison samples (e.g., variability or BH sphere of influence).

4. Neither barred galaxies, merging galaxies, nor galaxies with pseudo bulges seem to be significant outliers of the relation, at variance with recent predictions in the literature (Kormendy \& Ho 2013). However, this conclusion is based on small sample statistics and relies on low-resolution ground-based SDSS images which make a morphological classification challenging in the presence of a bright AGN point source. Larger samples with higher quality data are needed to further test any correlations between detailed host-galaxy morphology and $\mathrm{BH}$ mass.

In the next paper of this series we will discuss the other scaling relations, namely with spheroid luminosity and stellar mass as well as host-galaxy luminosity and stellar mass.

We thank the anonymous referee for valuable comments helping to improve the paper. VNB thanks Aaron Barth, Stéphane Courteau, Eric Emsellem, and Stefanie Komossa for discussions. VNB acknowledges assistance from a National Science Foundation (NSF) Research at Undergraduate Institutions (RUI) grant AST-1312296. Note that findings and conclusions do not necessarily represent views of the NSF. VNB and TT acknowledge support for program number HSTAR-12625.11-A, provided by NASA through a grant from the Space Telescope Science Institute, which is operated by the Association of Universities for Research in Astronomy, Incorporated, under NASA contract NAS5-26555. TT acknowledges support from the Packard Foundations in the form of a Packard Fellowship. DP acknowledges support through the EACOA Fellowship from The East Asian Core Observatories Association, which consists of the National Astronomical Observatories, Chinese Academy of Science (NAOC), the National Astronomical Observatory of Japan (NAOJ), Korean Astronomy and Space Science Institute (KASI), and Academia Sinica Institute of Astronomy and Astrophysics (ASIAA). JHW acknowledges support by the National Research Foundation of Korea (NRF) grant funded by the Korea government (2012-006087 and 2010-0027919). Data presented in this paper were obtained at the W. M. Keck Observatory, which is operated as a scientific partnership among Caltech, the University of California, and NASA. The Observatory was made possible by the generous financial support of the W. M. Keck Foundation. The authors recognize and acknowledge the very significant cultural role and reverence that the summit of Mauna Kea has always had within the indigenous Hawaiian community. We are most fortunate to have the opportunity to conduct observations from this mountain. This research has made use of the public archive of the Sloan Digital Sky Survey and the NASA/IPAC Extragalactic Database (NED) which is operated by the Jet Propulsion Laboratory, California Institute of Technology, under contract with the National Aeronautics and Space Administration.

Facilities: Keck:I (LRIS)

\section{REFERENCES}

Barth, A. J., Pancoast, A., Thorman, S. J. et al. 2011, ApJ, 743, L4

Becker, R. H., Helfand, D. J., White, R. L., Gregg, M., \&

Laurent-Muehleisen, S. A. 2003, VizieR On-line Data Catalog: VIII/71, http://cdsarc.ustrasbg.fr/viz-bin/Cat?VIII/71

Beifiori, A., Courteau, S., Corsini, E. M., \& Zhu, Y. 2012, MNRAS, 419, 2497

Bellovary, J., Holley-Bockelmann, K., Gültekin, K., Christensen, C., Governato, F., Brooks, A., Loebman, S., \& Munshi, F. 2014, MNRAS, 445,2667

Bennert, V. N., Treu, T., W. J.-H., Malkan, M. A., Le Bris, A., Auger, M. W. Gallagher, S., \& Blandford, R. D. 2010, ApJ, 708, 1507

Bennert, V. N., Auger, M. W., Treu, T., Woo, J.-H., \& Malkan, M. A. 2011a, ApJ, 726, 59 (Paper I)

Bennert, V. N., Auger, M. W., Treu, T., Woo, J.-H., \& Malkan, M. A. 2011b, ApJ, 742, 107

Bentz, M. C., Peterson, B. M., Pogge, R. W., Vestergaard, M., \& Onken, C. A. 2006, ApJ, 644, 133

Bentz, M. C., Peterson, B. M., Netzer, H., Pogge, R. W., \& Vestergaard, M. 2009, ApJ, 697, 160

Bentz, M. C. et al. 2013, ApJ, 767, 149

Boroson, T. A., \& Green, R. F. 1992, ApJS, 80, 109
Brewer, B. J., Treu, T., Pancoast, A., Barth, A. J., Bennert, V. N., Bentz, M C., Filippenko, A. V., Greene, J. E., Malkan, M. A., \& Woo, J.-H. 2011, ApJ, 733, L33

Capaccioli, M. 1989, in The World of Galaxies, ed. H. G. Corwin, L.

Bottinelli (Berlin: Springer-Verlag), 208

Ciotti, L., \& Ostriker, J. P. 2007, ApJ, 665, L5

Cisternas, M., Jahnke, K., Bongiorno, A., et al. 2011, ApJL, 741, 11

Courteau S., de Jong, R. S., \& Broeils, A. H. 1996, ApJ, 457, L73

Crenshaw, D. M., Kraemer, S. B., \& Gabel, J. R. 2003, AJ, 126, 1690

Croton, D. J. 2006, MNRAS, 369, 1808

Decarli, R., Falomo, R., Treves, A., Labita, M., Kotilainen, J. K., \& Scarpa,

R. 2010, MNRAS, 402, 2453

Deo, R. P., Crenshaw, D. M. \& Kraemer, S. B. 2006, AJ, 132, 321

de Vaucouleurs, G. 1948, Ann. d'Astrophys., 11, 247

Ferrarese, L., \& Ford, H. 2005, Space Science Reviews, 116, 3

Ferrarese, L., \& Merritt, D. 2000, ApJ, 539, L9

Ferrarese, L., Ford, H., \& Jaffe, W., 1996, ApJ, 470, 444

Gebhardt, K., et al. 2000, ApJ, 539, L13

Graham, A., W., 2007, MNRAS, 379, 711

Graham, A. W., 2008, ApJ, 680, 143

Graham, A., W., \& Li, 2009, ApJ, 698, 812 
Graham, A. W., \& Scott, N., 2013, ApJ, 764, 151

Graham, A. W., Onken, C. A., Athanassoula, E., \& Combes, F. 2011, MNRAS, 412,4

Greene, J. E., \& Ho, L. C. 2006, ApJ, 641, L21

Greene, J. E., \& Ho, L. C. 2007, ApJ, 667, 131

Greene, J. E., Ho, L. C., \& Barth, A. J. 2008, ApJ, 688, 159

Greene, J. E., Peng, C. Y., Kim, M., Kuo, C.-Y., Braatz, J. A., Impellizzeri, C. M.V., Condon, J. J., Lo, K. Y., Henkel, C. \& Reid, M. J. 2010, ApJ, 721,26

Gu, M., Chen, Z., \& Cao, X. 2009, MNRAS, 397, 3

Gültekin, K. et al. 2009, ApJ, 698, 198

Häring, N. \& Rix, H.-W. 2004, ApJ, 604, L89

Harris, C. E., Bennert, V. N., Auger, M. W., Treu, T., Woo, J.-H., \& Malkan, M. A. 2012, ApJS, 201, 29 (Paper II)

Herrnstein, J. R., Moran, J. M., Greenhill, L. J., \& Trotter, A. S. 2005, ApJ, 629, 719

Ho, L. C., \& Kim, M. 2014, ApJ, 789, 17

Hopkins, P. F. 2012, MNRAS, 420, 8

Hopkins, P. F., Hernquist, L., Cox, T. J., Robertson, B., \& Krause, E. 2007, ApJ, 669, 45

Hopkins, P. F., Hernquist, L., Cox, T. J., \& Keres, D. 2008, ApJS, 175, 356

Hu, J. 2008, MNRAS, 386, 2242

Hunt, L. K. \& Malkan, M. A. 1999, ApJ, 516, 660

Hunt, L. K. \& Malkan, M. A. 2004, ApJ, 616, 707

Jahnke, K., et al. 2009, ApJ, 706, 215

Jahnke, K. \& Maccio, A. 2011, ApJ, 734, 92

Kaspi, S., Maoz, D., Netzer, H., Peterson, B. M., Vestergard, M. \& Jannuzi, B. T. 2005, ApJ, 629, 61

Kaspi, S., Smith, P. S., Netzer, H., Maoz, D., Jannuzi, B. T. \& Giveon, U. 2000, ApJ, 533, 631

Kauffmann, G. \& Haehnelt, M. G. 2000, MNRAS, 311, 576

Kim, M., Ho, L. C., Peng, C. Y., Barth, A. J., \& Im, M. 2008, ApJS, 179, 283

Kormendy, J. 2001, The Seventh Texas-Mexico Conference on Astrophysics: Flows, Blows and Glows (Eds. William H. Lee and Silvia Torres-Peimbert), Revista Mexicana de Astronomía y Astrofísica, 10, 69-78

Kormendy, J., \& Richstone, D. 1995, ARA\&A, 33, 581

Kormendy, J., \& Kennicutt, R. C., Jr. 2004, ARA\&A, 42, 603

Kormendy, J. \& Bender, R. 2011, Nature, 469, 377-380

Kormendy, J. \& Ho, L. C. 2013, ARAA, 51

Kormendy, J., Bender, R., \& Cornell, M. E. 2011, Nature, 469, 374

Koss, M., Mushotzky, R., \& Veilleux, S. et al. 2011, ApJ, 739, 57

Kuo, C. Y. et al. 2011, ApJ, 727, 20

Läsker, R., Ferrarese, L., van de Ven, G., \& Shankar, F. 2014, ApJ, 780, 70

Lintott, C., Schawinski, K., Bamford, S., Slosar, A., Land, K., Thomas, D.

Edmondson, E., Masters, K., Nichol, R. C., Raddick, M. J., Szalay, A.,

Andreescu, D., Murray, P., \& Vandenberg, J. 2011, MNRAS, 410, 166

MacKenty, J. W. 1990, ApJS, 72, 231

Magorrian, J., et al. 1998, AJ, 115, 2285

Malkan, M. A., Gorjian, V., \& Tam, R. 1998, ApJS, 117, 25

Marconi, A., \& Hunt, L. K. 2003, ApJ, 589, L21

Marconi, A. et al. 2001, ApJ, 549, 915

McConnell, N. J. \& Ma, C.-P. 2013, ApJ, 764, 184

McConnell, N. J., Ma, C.-P., Gebhardt, K., Wright, S. A., Murphy, J. D.,

Lauer, T. R., Graham, J. R., \& Richstone, D. O. 2011, Nature, 480, 215

McGill, K. L., Woo, J.-H., Treu, T., \& Malkan, M. A. 2008, ApJ, 673, 703

McLure, R. J., Jarvis, M. J., Targett, T. A., Dunlop, J. S. \& Best, P. N. 2006, MNRAS, 368, 1395

Merloni, A., et al. 2010, ApJ, 708, 137

Merritt, D., \& Ferrarese, L. 2001, ApJ, 547, 110

Nowak N., Thomas, J., Erwin, P., Saglia, R. P., Bender, R., \& Davies, R. I. 2010, MNRAS, 403, 646
Onken, C. A., Ferrarese, L., Merritt, D., Peterson, B. M., Pogge, R. W., Vestergaard, M., \& Wandel, A. 2004, ApJ, 615, 645

Pancoast, A., Brewer, B. J., \& Treu, T. 2011, ApJ, 730, 139

Pancoast, A., et al. 2012, ApJ, 754, 49

Pancoast, A., Brewer, B. J., Treu, T., et al. 2014, MNRAS, 445, 3073

Park, D., Woo, J.-H., Bennert, V. N., Treu, T., Auger, M. W., \& Malkan, M

A. 2015, ApJ, 799, 164

Park, D., Kelly, B. C., Woo, J.-H., \& Treu, T. 2012, ApJS, 203, 6

Parry, O. H., Eke, V. R., \& Frenk, C. S. 2009, MNRAS, 396, 1972

Peng, C. Y. 2007, ApJ, 671, 1098

Peng, C. Y., Ho, L. C., Impey, C. D., \& Rix, H.-W. 2002, AJ, 124, 266

Peng, C. Y., Impey, C. D., Ho, L. C., Barton, E. J., \& Rix, H.-W. 2006a, ApJ, 640,114

Peng, C. Y., Impey, C. D., Rix, H.-W., Kochanek, C. S., Keeton, C. R.,

Falco, E. E., Lehár, J., \& McLeod, B. A. 2006b, ApJ, 649, 616

Prugniel, P. \& Simien, F. 1997, A\&A, 321, 111

Rafter, S. E., Crenshaw, D. M. \& Wiita, P. J. 2009, AJ, 137,

Riechers, D. A., Walter, F., Brewer, B. J., Carilli, C. L., Lewis, G. F., Bertoldi, F., \& Cox, P. 2008, ApJ, 686, 851

Riechers, D. A., Walter, F., Carilli, C. L., \& Lewis, G. F. 2009, ApJ, 690, 463

Robertson, B., Hernquist, L., Cox, T. J., Di Matteo, T., Hopkins, P. F., Martini, P. \& Springel, V. 2006, ApJ, 641, 90

Salviander, S., Shields, G. A., Gebhardt, K., \& Bonning, E. W. 2007, ApJ, 662,131

Sani, E., Marconi, A., Hunt, L. K., \& Risaliti, G., 2011, MNRAS, 413, 1479

Schramm, M. \& Silverman, 2013, ApJ, 767, 13

Schulze, A. \& Wisotzki, L., 2014, MNRAS, 438, 3422

Shen, J., Vanden Berk, D. E., Schneider, D. P., \& Hall, P. B. 2008, AJ, 135 928

Shields, G. A., Menezes, K. L., Massart, C. A., \& Vanden Bout, P. 2006, ApJ, 641, 683

Simard, L., Mendel, J. T., Patton, D. R., Ellison, S. L., \& McConnachie, A. W. 2011, ApJS, 196, 11

Tremaine, S. et al. 2002, ApJ, 574, 740

Treu, T., Malkan, M. A., \& Blanford, R. D. 2004, ApJ, 615, L97

Treu, T., Woo, J.-H., Malkan, M. A., \& Blanford, R. D. 2007, ApJ, 667, 117

Valdes, F., Gupta, R., Rose, J. A., Singh, H. P., \& Bell, D. J. 2004, ApJS, 152,251

van der Marel, R. P. 1994, MNRAS, 270, 271

van der Marel, R. P. \& Franx, M. 1993, ApJ, 407, 525

van der Marel, R. P., Cretton, N., de Zeeuw, P. T., \& Rix, H. 1998, ApJ, 493 729

Vestergaard, M. 2002, ApJ, 571, 733

Vestergaard, M. \& Peterson, B. M. 2006, ApJ, 641, 689

Volonteri, M., \& Stark, D. P. 2011, MNRAS, 417, 2085

Volonteri, M., Haardt, F., \& Madau, P. 2003, ApJ, 582, 559

Walter, F., Carilli, C., Bertoldi, F., Menten, K., Cox, P., Lo, K. Y., Fan, X., \&

Strauss, M. A. 2004, ApJ, 615, L17

Wandel, A., Peterson, B. M., \& Malkan, M. A. 1999, ApJ, 526, 579

Weiss, A., Downes, D., Neri, R., Walter, F., Henkel, C., Wilner, D. J., Wagg, J. \& Wiklind, T. 2007, A\&A, 467, 955

Woo, J.-H., \& Urry, C. M. 2002, ApJ, 579, 530

Woo, J.-H., Treu, T., Malkan, M. A., \& Blandford, R. D. 2006, ApJ, 645, 900

Woo, J.-H., Treu, T., Malkan, M. A., \& Blandford, R. D. 2008, ApJ, 681, 925

Woo, J.-H., et al. 2010, ApJ, 716, 269

Woo, J.-H., et al. 2013, ApJ, 716, 269

Woo, J.-H., Yoon, Y., Park, S., Park, D., \& Kim, S. C. 2015, ApJ, 801, 1

APPENDIX

\section{A. BROAD H $\beta$ EMISSION-LINE FITTING}

Figs. A1 and A2 show fits to the broad $\mathrm{H} \beta$ emission line for 79 objects for which we were able to measure $M_{\mathrm{BH}}$. 


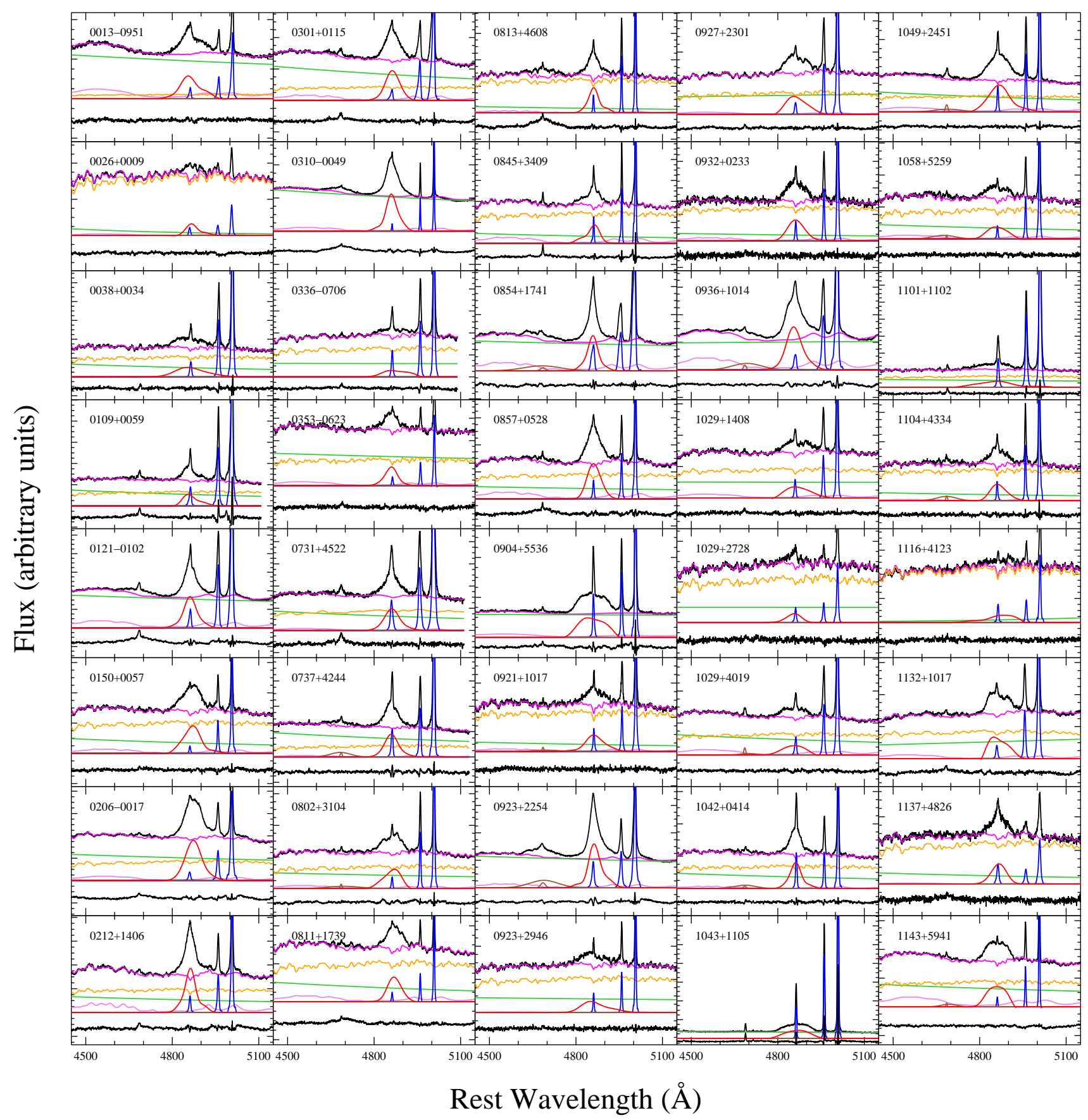

FIG. A1. - Determination of the second moment of the broad $\mathrm{H} \beta$ emission using a multi-component spectral decomposition. In the upper region, the observed spectrum is shown in black with the best-fit model consisting of the continuum, FeII, and host galaxy starlight in magenta. Below, the best-fit to the power-law continuum is shown in green with the stellar spectrum in yellow. Further below are the narrow lines of $\mathrm{H} \beta \lambda 4861$, and [OIII] $\lambda \lambda 4959,5007$ in blue, the broad and narrow components of HeII $\lambda 4686$ in brown (only included if blended with the broad $\mathrm{H} \beta$ ), the broad component of $\mathrm{H} \beta$ in red and the FeII contribution in purple. Finally, the residuals are shown in black (arbitrarily shifted downward for clarity).

\section{B. NOTES ON INDIVIDUAL OBJECTS}

One object in our sample, $1223+0240$ also known as MRK50, is also in the reverberation-mapped AGN sample and its BH mass has been estimated both in a traditional way (Barth et al. 2011), $\log M_{\mathrm{BH}} / M_{\odot}=7.51 \pm 0.05$ (and that is also the value used when plotting the reverberation-mapped AGNs; note that the quoted uncertainty does not include the uncertainty on the virial factor) as well as using dynamical modeling (Pancoast et al. 2012), $\log M_{\mathrm{BH}} / M_{\odot}=7.57_{-0.27}^{+0.44}$. Both values are within the uncertainties of the $\mathrm{BH}$ mass determined here via the virial method of $\log M_{\mathrm{BH}} / M_{\odot}=7.25 \pm 0.4$. Pancoast et al. (2012) infer that the geometry of the BLR is a nearly face-on thick disk, with a potential net inflow or outflow. MRK50 is bulge dominated (MacKenty 1990; Koss et al. 2011) and Ho \& Kim (2014) classify its bulge as classical bulge which matches our classification. Note that Fig. 2 includes MRK50 twice, once amongst the reverberation-mapped AGNs with the reverberation-mapped MBH, 


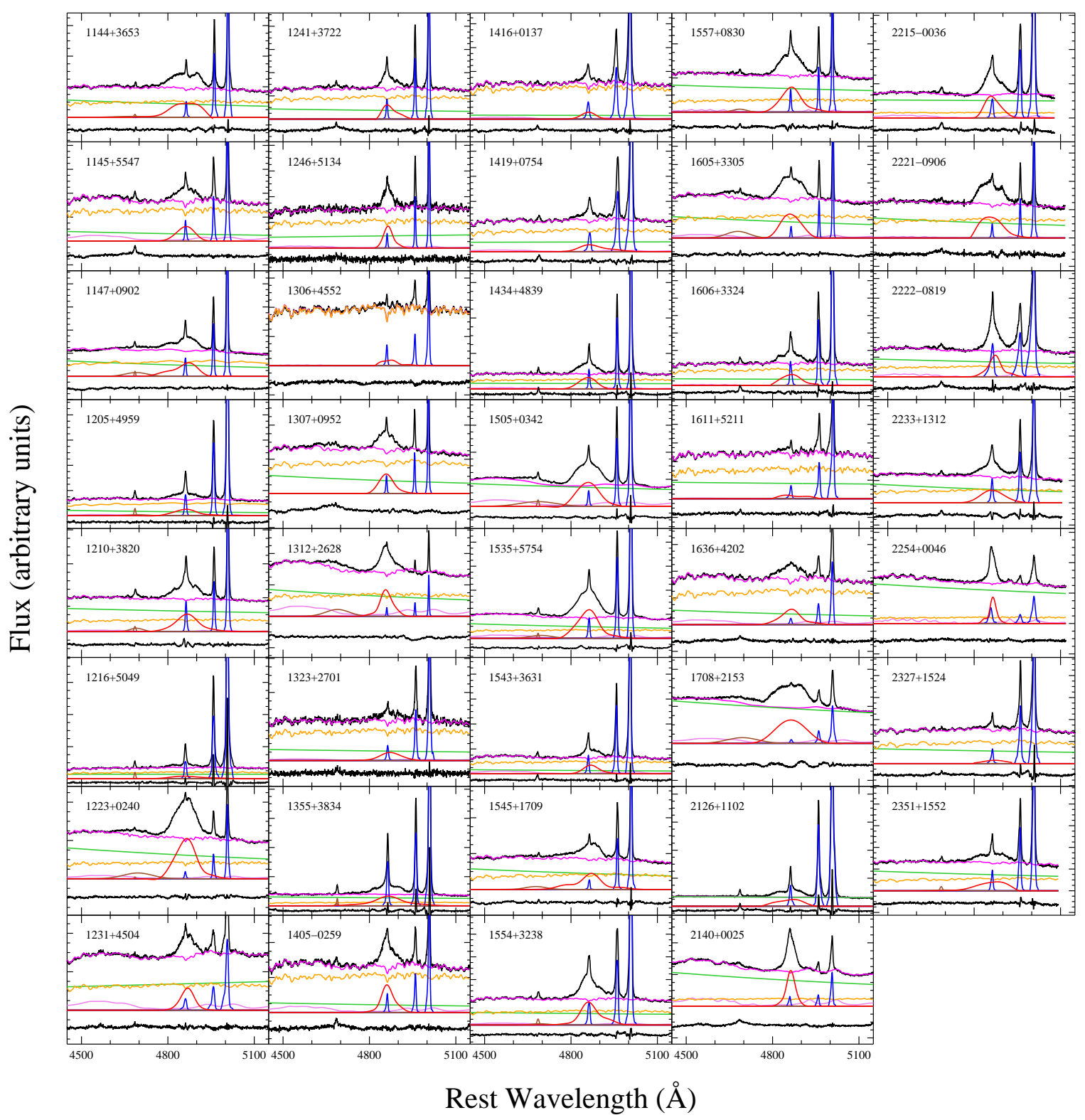

FIG. A2.- Fig. A1 continued

once amongst our local sample.

1535+5754, aka MRK290, was included in the Lick AGN Monitoring Program (LAMP 2008), but did not exhibit strong variations, so no BH mass was derived. Bentz et al. (2013) classify the host galaxy as an early-type spiral galaxy (Sa-Sab) at a relatively low inclination, based on HST images. However, this classification is questionable. Deo et al. (2006) describe the host galaxy as "probably elliptical", but mistakenly classified as unbarred spiral previously (Crenshaw et al. 2003). We classified MRK290 as elliptical galaxy with a classical bulge.

1216+5049 is known as MRK1469 and was classified as a highly inclined spiral galaxy with only large-scale dust lanes visible in Deo et al. (2006), in agreement with our classification. 